TRANSACTIONS OF THE

AMERICAN MATHEMATICAL SOCIETY

Volume 352, Number 4, Pages 1889-1912

S 0002-9947(99)02479-4

Article electronically published on November 17, 1999

\title{
LOCAL PRODUCT STRUCTURE FOR EQUILIBRIUM STATES
}

\author{
RENAUD LEPLAIDEUR
}

\begin{abstract}
The usual way to study the local structure of Equilibrium State of an Axiom-A diffeomorphism or flow is to use the symbolic dynamic and to push results on the manifold. A new geometrical method is given. It consists in proving that Equilibrium States for Hölder-continuous functions are related to other Equilibrium States of some special sub-systems satisfying a sort of expansiveness. Using different kinds of extensions the local product structure of Gibbs-measure is proven.
\end{abstract}

\section{INTRODUCTION}

In [5, Bowen and Marcus define a notion of the transversal to a $n$-dimensional foliation $\mathcal{G}$ in a metric space $X$ and a notion of a $\mathcal{G}$-invariant measure. They prove that up to a constant there is a unique $\mathcal{G}$-invariant measure for any Axiom-A diffeomorphism or flow where $\mathcal{G}$ is the strong stable or unstable foliation for any basic set. In [8], for the Axiom-A flow case $(\Omega, \Phi)$, Haydn proves the existence of a transversal system of measure $\left\{\mu_{x}\right\}$ supported on the local weak unstable manifold which is not invariant along the $W^{s s}$-foliation but which admits a Jacobian of the type $e^{\omega_{x, x^{\prime}}}$, with $\omega_{x, x^{\prime}}=\int_{0}^{\infty}\left(F \circ \Phi_{s} \circ \rho_{x, x^{\prime}}-F \circ \Phi_{s}\right) d s$ where $F$ is any Höldercontinuous function $\Omega \rightarrow \mathbb{R}$. In his proof, Haydn assumes that $\Phi_{s *} \mu_{x}$ and $\mu_{\Phi_{s}(x)}$ are two equivalent measures and uses the symbolic dynamic just as in [5. In [1], Babillot and Ledrappier prove the previous result, plus uniqueness, without assuming that $\Phi_{s *} \mu_{x}$ and $\mu_{\Phi_{s}(x)}$ are absolutely continuous. Unfortunately, they still use the symbolic dynamic, which prevents us from extending this to the nonuniform dynamic. Another proof of this result is given here. As we don't have uniqueness, it is in one sense weaker than in 11, but it is more geometrical and doesn't hide all the real dynamic on the manifold as the symbolic dynamic does. It should be easier to extend it to the non-uniform dynamic case.

The proof is based on the definition of a sub-dynamical system which is related to the "big" one. For this sub-system, we prove existence and uniqueness of Equilibrium States for good potentials and we connect them to the Equilibrium States for the global system. As we are looking for measures, we use a Perron-Frobénius operator acting on continuous functions to define its adjoint acting on the set of probability measures. This makes the proof more complicated but is necessary. We are then able to recognize among these states a good candidate for the transversal measures. Moreover, our proof gives the local product structure of the Gibbs

Received by the editors June 30, 1997.

2000 Mathematics Subject Classification. Primary 37D20, 37D35.

(C)2000 American Mathematical Society 
measures and, as a corollary, the pointwise dimension formula. Theorems are announced and proved for Axiom-A diffeomorphisms but can easily be extended for Axiom-A flows which are suspended flows.

To be complete, some well-known facts about Axiom-A theory and extensions are recalled at the end.

As we intend to later extend these results to the non-uniform hyperbolic case, we have taken care to use in our proofs only facts about Axiom-A that can be in some way extended to the general case.

\section{BACKGROUND AND RESUltS}

2.1. First definitions. Let $M$ be a compact Riemannian manifold, and let $f$ : $M \rightarrow M$ be a $C^{1+\alpha}$ mixing diffeomorphism, with the Axiom-A property. We assume that a metric adapted to $f$ has been chosen such that the following holds (see [4] for such metric)

(1) Periodic points are dense in the set of non-wandering points $\Omega$.

(2) $\Omega$ is hyperbolic, meaning that for all $x$ in $\Omega$ there is a continuous splitting of the tangent space $T_{x} M$ with

(a) $T_{x} M=E_{x}^{u} \oplus E_{x}^{s}$;

(b) $d f_{x}\left(E_{x}^{u}\right)=E_{f(x)}^{u}$ and $d f_{x}\left(E_{x}^{s}\right)=E_{f(x)}^{s}$;

(c) there exists $\lambda \in] 1,+\infty[$ such that for all integer $n \geq 0$.

$$
\begin{aligned}
\left\|d f_{x}^{n}(v)\right\| & \leq \frac{1}{\lambda^{n}}\|v\| \text { for all } v \in E_{x}^{s}, \\
\left\|d f_{x}^{-n}(v)\right\| & \leq \frac{1}{\lambda^{n}}\|v\| \text { for all } v \in E_{x}^{u} .
\end{aligned}
$$

$\varepsilon_{0}$ will denote an expansiveness constant and we will denote respectively $W_{\varepsilon_{0}}^{s}(x)$ and $W_{\varepsilon_{0}}^{u}(x)$ by $W_{l o c}^{s}(x)$ et $W_{l o c}^{u}(x)$. If $\varepsilon_{0}$ is small enough, then

$$
\begin{aligned}
{[., .]:\{(x, y) \in \Omega \times \Omega: d(x, y) \leq \rho\} } & \longrightarrow \Omega, \\
x & \longmapsto[x, y] \stackrel{\text { def }}{=} W_{l o c}^{s}(x) \cap W_{l o c}^{u}(y)
\end{aligned}
$$

is well defined for all $\rho \leq \rho_{0}$, where $\rho_{0}$ is sufficiently small. In [4], Bowen shows that there exists a finite Markov partition with diameter as small as we want, such that each atom of the partition is a proper rectangle. We pick a proper rectangle $R$ with the Markov property and with diameter smaller than $\varepsilon\left(4 \varepsilon \leq \rho_{0}\right.$, and $\left.4 \varepsilon<\varepsilon_{0}\right)$.

Let us assume that $\mathcal{A}$ is an $\alpha$-Hölder-continuous function from $\Omega$ to $\mathbb{R}$. In the set of invariant probability measures there is one particular measure, called the Equilibrium State for the potential $\mathcal{A}$. It is denoted by $\mu^{\mathcal{A}}$, and it is the unique $f$-invariant measure which realizes equality in

$$
h_{\mu^{\mathcal{A}}}(f)+\int \mathcal{A} d \mu^{\mathcal{A}}=\sup \left\{h_{\nu}(f)+\int \mathcal{A} d \nu\right\},
$$

where the supremum is taken over the set of invariant probability measures. We will denote by $\mathcal{P}_{\mathcal{A}}$ this supremum.

Let us make precise here the definition of a transversal to the stable foliation (see [5]).

Definition 2.1. A transversal to the stable foliation $W^{s}(\Omega)$ at $x$ is a compact $K$ containing $x$ such that there exists a one-to-one map $\Phi: K \times D^{s} \rightarrow \Omega$ with $\Phi\left(y \times D^{s}\right) \subset W^{s}(y), \Phi(y, 0)=y$ for any $y$ and $\Phi\left(K \times D^{s}\right)$ being a neighborhood of $x$ in $\Omega$. 
If $K$ is a transversal to the stable foliation, a transversal measure $\mu_{K}$ on $K$ will be a finite nonnegative Borel measure on $K$, and a system of transversal measures will be a family $\left\{\mu_{K}\right\}_{K \text { transversal }}$ of transversal measures on all transversals $K$. If $K$ and $K^{\prime}$ are two transversals, two sets $A \subset K$ and $A^{\prime} \subset K^{\prime}$ will be called $W^{s}$-conjugate if there is a one-to-one Borel map $\pi_{A, A^{\prime}}: A \rightarrow A^{\prime}$ such that, for all $x$ in $A, \pi_{A, A^{\prime}}(x) \in W^{s}(x)$.

Definition 2.2. For $\omega: \Omega \times \Omega \rightarrow \mathbb{R}$, we will say that a system of transversal measures is $\omega$-absolutely continuous ( $\omega$-a.c.) if and only if

(1) for any transversals $K$ and $K^{\prime}$, for any $W^{s}$-conjugate sets $A \subset K$ and $A^{\prime} \subset K^{\prime}$, $\mu_{K^{\prime}}\left(A^{\prime}\right)=\int_{A} \omega\left(\pi_{A, A^{\prime}}(x), x\right) d \mu_{K}(x) ;$

(2) $\mu_{K}(K)>0$ for one $K$.

2.2. Results. Let $x$ and $x^{\prime}$ be two points in $\Omega$ such that $x^{\prime} \in W^{s}(x)$. We set

$$
w\left(x, x^{\prime}\right)=\sum_{k=0}^{+\infty} \mathcal{A} \circ f^{k}\left(x^{\prime}\right)-\mathcal{A} \circ f^{k}(x) .
$$

Theorem 2.3 (A). There exists a unique $W^{s}$-transversal measure system $\left\{\mu_{K}\right\}$ such that

(i) $\left\{\mu_{K}\right\}$ is $e^{w}-$ a.c.;

(ii) $\frac{d \mu_{f(K)} f(x)}{d f_{*} \mu_{K}(x)}=e^{\mathcal{A}(x)-\mathcal{P}_{\mathcal{A}}}$.

This transversal system is equivalent on each $W_{l o c}^{u}(x)$ to the conditional measure $\mu^{\mathcal{A}, u}$ of the Equilibrium State $\mu^{\mathcal{A}}$ with respect to any measurable partition subordinate to the unstable foliation.

We refer the reader to [1] and 13] for precise definitions about measurable partitions and subordinate partitions. The proof of Theorem A needs the absolute continuity of the conditional measures of the Equilibrium State. We shall first prove this as well as a result on the existence of pointwise dimension.

Definition 2.4. Let $\nu$ be a probability measure on the metric space $\mathcal{X}$. We define the upper pointwise dimension (respectively lower) of $\nu$ at a point $x_{0}$ as the real number

$$
\bar{\delta}\left(x_{0}, \nu\right) \stackrel{\text { def }}{=} \limsup _{\varepsilon \rightarrow 0} \frac{\log \nu\left(B\left(x_{0}, \varepsilon\right)\right)}{\log \varepsilon}
$$

(resp. $\underline{\delta}\left(x_{0}, \nu\right) \stackrel{\text { def }}{=} \lim \inf \ldots$ ). If these two numbers are equal, we call their common value the pointwise dimension of $\nu$ at $x_{0}$ and we denote it by $\delta\left(x_{0}, \nu\right)$.

Theorem $2.5\left(\mathbf{A}^{\prime}\right)$. The Equilibrium State has local product structure

$$
d \mu^{\mathcal{A}}([y, z])=\varphi_{x}(y, z) d \mu_{x}^{\mathcal{A}, u}(y) \otimes d \mu_{x}^{\mathcal{A}, s}(z)
$$

where $\mu_{x}^{\mathcal{A}, u}$ and $\mu_{x}^{\mathcal{A}, s}$ denote the conditional measures of $\mu^{\mathcal{A}}$ with respect to any measurable partition subordinate to the unstable and the stable foliation; $y$ is any point in $W_{\text {loc }}^{u}(x)$ and $z$ is any point in $W_{l o c}^{s}(x)$, and $\varphi_{x}$ is a non-negative Borel function.

Moreover, $\mu^{\mathcal{A}}, \mu_{x}^{\mathcal{A}, u}$ and $\mu_{x}^{\mathcal{A}, s}$ have pointwise dimensions, $\delta, \delta^{u}$ and $\delta^{s}, \mu^{\mathcal{A}}$ almost everywhere constant, and

$$
\delta=\delta^{u}+\delta^{s}
$$


Remark. This last equality $\delta=\delta^{u}+\delta^{s}$ is a particular case of a general fact in the non-uniform hyperbolic case that has just been proved in [2].

\section{The Dynamical System $\left(F, g_{F}\right)$}

3.1. Definitions. Pick $R$ one particular proper rectangle with the Markov property. By Poincaré's theorem, we define respectively $R_{n}$ and $R_{\infty}$ as the set of points in $R$ returning at least $n$ times in $R$ by the map $f$ and the set of points returning infinitely often. The first return time map from $R$ to $\mathbb{N}$ is defined by

$$
r(x)=\left\{\begin{array}{cl}
0 & \text { if } x \notin R_{1}, \\
k_{0} & \text { if } k_{0}=\inf \left\{k \in \mathbb{N} \text { s.t. } f^{k}(x) \in R\right\}<+\infty .
\end{array}\right.
$$

Then we define the map $g$ by

$$
\begin{aligned}
g: \quad R & \longrightarrow R, \\
x & \longmapsto g(x) \stackrel{\text { def }}{=} f^{r(x)}(x) .
\end{aligned}
$$

For any integer $n$, and for any $x$ in $R_{n}$, we write $r^{n}(x) \stackrel{\text { def }}{=} r^{n-1}(x)+r\left(f^{r^{n-1}(x)}(x)\right)$.

Definition 3.1. Every $W^{u}(x, R)$ (resp. $\left.W^{s}(x, R)\right)$, where $x \in R$, is called an unstable leaf (resp. stable) of $R$.

If $F$ is an unstable leaf of $R$, we denote $F_{1} \stackrel{\text { def }}{=} F \cap R_{1}, F_{\infty} \stackrel{\text { def }}{=} F \cap R_{\infty}$, and $\partial F \stackrel{\text { def }}{=} F \cap \partial^{s} R$, where $\partial^{s} R$ is the stable topologic boundary of $R$.

We also define the stable holonomy onto $F$ by

$$
\begin{aligned}
\pi_{F}: R & \longrightarrow F, \\
x & \longmapsto \pi_{F}(x) \stackrel{\text { def }}{=} W^{s}(x, R) \cap F .
\end{aligned}
$$

We can easily check that we have:

Lemma 3.2. Every unstable leaf of $R$ is a compact set.

In the whole paper, $F$ will denote an unstable leaf of $R \backslash \partial^{u} R$.

We define the map $g_{F}: F \longrightarrow F$ by $x \longmapsto g_{F}(x) \stackrel{\text { def }}{=} \pi_{F} \circ g(x)$, and we denote by $H_{1}$ the set $F \backslash F_{1}$.

The Markov property of $R$ implies a similar property for the system $\left(F, g_{F}\right)$ on the inverse branches.

Definition 3.3. We call $n$-cylinder $\left(n \in \mathbb{N}^{*}\right)$ of $F$ every $f^{-r^{n}(x)} W^{u}\left(f^{r^{n}(x)}(x), R\right)$ with $x$ in $R_{n}$ and $f^{r^{n}(x)}(x) \in \operatorname{int}\left(W^{u}\left(f^{r^{n}(x)}(x), R\right)\right)$. The integer $n$ is called order of the cylinder.

Some of the points may be in several cylinders of same order. If we denote by $\partial_{g} F$ the set $\bigcup_{n \in \mathbb{N}} g_{F}^{-n}(\partial F)$, we claim that a point, which is in several cylinders of the same order, must be in $\partial_{g} F$. We also remark that the Markov property implies that $g_{F}(\partial F) \subset \partial F$.

Cylinders pretend to be the inverse branches, but because of the existence of the sets $H_{1}$ and $\partial_{g} F, g_{F}^{n}$ doesn't really map any $n$-cylinder to $F$. Part of the job will consist in studying these bad sets $H_{1}$ and $\partial_{g} F$. Let us start by giving some definitions.

Definition 3.4. If $C$ is a 1-cylinder of $F$, we call return time of $C$ the common return time of every point in $C \backslash \partial_{g} F$. 
Definition 3.5. Let $x \in F$. We say a point $y$ of $F$ is a 1-preimage of $x$ by $g_{F}$ if and only if

(i) $y \in F_{1}$;

(ii) there exists a 1-cylinder containing $y$, with return time $r(y, C)$, such that $\pi_{F} \circ f^{r(y, C)}(y)=x$.

We will denote by $\operatorname{Ant}(x)$ the set of 1 -preimages of $x$ and by $\operatorname{Ant}(k, x)$ the subset of $\operatorname{Ant}(x)$ of points $y$ such that $\pi_{F} \circ f^{k}(y)=x$.

If $k \in \mathbb{N}^{*}$, we define the set of $k$-preimages of $x$ by $g_{F}, A n t_{k}(x)$, by

$$
\operatorname{Ant}_{k}(x) \stackrel{\text { def }}{=} \operatorname{Ant}\left(\operatorname{Ant}_{k-1}(x)\right) .
$$

Remark. Points in $A_{n}(x)$ are points in $k$-cylinders.

Using the mixing property we can easily check that:

Lemma 3.6. For all $x$ in $F, \bigcup_{n \in \mathbb{N}} A n t_{n}(x)$ is dense in $F$.

3.2. The metric $\eta$. The natural metric on $F$ doesn't separate enough points. For instance points can be very close in $F$ but in different cylinders. We introduce a quasi-metric, which is more adapted to the cover by cylinders. To keep control on backward and forward orbits we look at the half-time we need to be greater than an expansivity constant.

Definition 3.7. Let $x$ and $y$ be in the same unstable leaf of $R$; we call $n(x, y)$ the greatest positive integer such that

$$
d\left(f^{k}(x), f^{k}(y)\right) \leq \varepsilon \quad \forall k \leq n(x, y) .
$$

We denote by $N(x, y)=\left[\frac{1}{2} n(x, y)\right]+1$, where $[x]$ is the integer part of the real number $x$.

Let us now show that the map $N(.,$.$) is almost constant along the stable$ foliation.

Lemma 3.8. There exists an integer $P(R)$ such that for all $x$ and $y$ in $F$ and all $x^{\prime}$ and $y^{\prime}$ satisfying $x^{\prime} \in W^{s}(x, R), y^{\prime} \in W^{s}(y, R)$, and $x^{\prime} \in W^{u}\left(y^{\prime}, R\right)$, we have

$$
N(x, y)-P(R) \leq N\left(x^{\prime}, y^{\prime}\right) \leq N(x, y)+P(R) .
$$

Proof. Let $p \in \mathbb{N}$ such that $d\left(f^{p}\left(x^{\prime}\right), f^{p}\left(y^{\prime}\right)\right)<\varepsilon$ and $d\left(f^{p+1}\left(x^{\prime}\right), f^{p+1}\left(y^{\prime}\right)\right)>\varepsilon$. Contraction on stable manifolds leads to $d\left(f^{p}(x), f^{p}\left(x^{\prime}\right)\right)<\varepsilon$ and $d\left(f^{p}(y), f^{p}\left(y^{\prime}\right)\right)<$ $\varepsilon$, which implies $d\left(f^{p}(x), f^{p}\left(y^{\prime}\right)\right)<2 \varepsilon$. The $\gamma$-Hölder-continuous property of [., .] proves that

$$
\left(d\left(f^{p}\left(y^{\prime}\right), f^{p}\left(x^{\prime}\right)\right)\right)^{\frac{1}{\gamma}} \leq d\left(f^{p}(y), f^{p}(x)\right) \leq\left(d\left(f^{p}\left(y^{\prime}\right), f^{p}\left(x^{\prime}\right)\right)\right)^{\gamma} .
$$

We know that there exists $\lambda^{\prime}$ such that $d\left(f^{p}\left(y^{\prime}\right), f^{p}\left(x^{\prime}\right)\right)>\varepsilon / \lambda^{\prime}$ since $p=n\left(x^{\prime}, y^{\prime}\right)$, and it is sufficient to have $\lambda^{q}\left(\varepsilon / \lambda^{\prime}\right)^{\frac{1}{\gamma}}>\varepsilon$ to get $d\left(f^{p+q}(x), f^{p+q}(y)\right)>\varepsilon$. This gives

$$
n(x, y) \leq n\left(x^{\prime}, y^{\prime}\right)+\frac{(\gamma-1) \log \varepsilon+\log \lambda^{\prime}}{\gamma \log \lambda} .
$$

The other inequality follows from the same computation by exchanging $(x, y)$ and $\left(x^{\prime}, y^{\prime}\right)$.

A quasi-metric on $F$ is defined by the following. 
Definition 3.9. Let $x$ and $y$ be in $F$; we define

$$
\eta(x, y) \stackrel{\text { def }}{=} \frac{1}{\lambda^{\alpha \gamma N(x, y)}} .
$$

Remark. There exists a universal constant $C$ such that $d^{\alpha}(x, y) \leq C \eta(x, y)$.

Associated to this quasi-metric a set of Hölder-continuous functions is defined.

Definition 3.10. We denote by $C_{\eta}^{0}(F)$ the set of continuous functions from $F$ to $\mathbb{R}$ such that

$$
C_{\phi} \stackrel{\text { def }}{=} \sup _{\substack{x, y \\ x \neq y}} \frac{|\phi(x)-\phi(y)|}{\eta(x, y)}<+\infty .
$$

We define a norm $\|\cdot\|_{\eta}: C_{\eta}^{0}(F) \rightarrow \mathbb{R}^{+}$by $\phi \mapsto C_{\phi}+\|\phi\|_{\infty}$.

Then we have.

Proposition 3.11. $\left(C_{\eta}^{0}(F),\|\cdot\|_{\eta}\right)$ is a Banach space.

3.3. New potential. We want to see $(\Omega, f)$ as an extension of $\left(F, g_{F}\right)$ and the measure $\mu^{\mathcal{A}}$ as an extension of an Equilibrium State for $\left(F, g_{F}\right)$. To that aim, we have to exhibit a new potential, related to $\mathcal{A}$ but taking care of the fact that we are iterating $g_{F}$ and not $f$. We define functions $\mathcal{B}^{\prime}$ and $\omega$ on $F \backslash\left(H_{1} \cup \partial_{g} F\right)$ by

$$
\begin{aligned}
\mathcal{B}^{\prime}(x) & \stackrel{\text { def }}{=} \sum_{k=0}^{r(x)-1} \mathcal{A} \circ f^{k}(x) \text { and } \\
\omega(x) & \stackrel{\text { def }}{=} \sum_{k=0}^{+\infty} \mathcal{A} \circ f^{k+r(x)}(x)-\mathcal{A} \circ f^{k} \circ \pi_{F} \circ f^{r(x)}(x) .
\end{aligned}
$$

The function $\mathcal{B}$ defined by $\mathcal{B}(x) \stackrel{\text { def }}{=} \mathcal{B}^{\prime}(x)+\omega(x)$ provides a new potential on $F \backslash\left(H_{1} \cup \partial_{g} F\right)$. Using $\mathcal{B}$ means we are iterating $g$ and not $f$, and $\omega$ introduces the drift coming from the iteration of $g_{F}$ and not $g$. Baire's theorem shows that $\partial_{g} F$ has empty interior, and $\mathcal{B}^{\prime}, \omega$ and $\mathcal{B}$ can be extended by continuity in every cylinder to $F \backslash H_{1}$. As points in $\partial_{g} F$ can be in several 1-cylinders, we will write $\mathcal{B}\left(x, C_{i}\right), \omega\left(x, C_{i}\right)$ and $\mathcal{B}\left(x, C_{i}\right)$ where $C_{i}$ is a 1-cylinder containing $x \in F \backslash H_{1}$.

Lemma 3.12. Let $x$ and $y$ be two points in $F$ and $x^{\prime}$ and $y^{\prime}$ two 1-preimages of $x$ and $y$ from the same 1-cylinder, $C_{i}$. There exists a universal constant $C$ such that

$$
\left|\omega\left(x^{\prime}, C_{i}\right)-\omega\left(y^{\prime}, C_{i}\right)\right| \leq C \eta(x, y) .
$$

Proof. Let $r$ be the return time of the cylinder $C_{i}$ and $N=N(x, y)$. We have

$$
\begin{aligned}
\omega\left(x^{\prime}, C_{i}\right)-\omega\left(y^{\prime}, C_{i}\right)= & \sum_{k=0}^{N} \mathcal{A} \circ f^{k+r}\left(x^{\prime}\right)-\mathcal{A} \circ f^{k+r}\left(y^{\prime}\right) \\
& -\sum_{k=0}^{N} \mathcal{A} \circ f^{k} \circ \pi_{F} \circ f^{r}\left(x^{\prime}\right)-\mathcal{A} \circ f^{k} \circ \pi_{F} \circ f^{r}\left(y^{\prime}\right) \\
& +\sum_{k=N+1}^{+\infty} \mathcal{A} \circ f^{k+r}\left(x^{\prime}\right)-\mathcal{A} \circ f^{k} \circ \pi_{F} \circ f^{r}\left(x^{\prime}\right) \\
& -\sum_{k=N+1}^{+\infty} \mathcal{A} \circ f^{k+r}\left(y^{\prime}\right)-\mathcal{A} \circ f^{k} \circ \pi_{F} \circ f^{r}\left(y^{\prime}\right) .
\end{aligned}
$$


- upper bound of the first term: Using Lemma (3.8), we check that this term is less than $\sum_{k=0}^{N^{\prime}+p}\left|\mathcal{A} \circ f^{k+r}\left(x^{\prime}\right)-\mathcal{A} \circ f^{k+r}\left(y^{\prime}\right)\right|$. The Hölder property of $\mathcal{A}$ and the expanding property imply that

$$
\sum_{k=0}^{N^{\prime}}\left|\mathcal{A} \circ f^{k+r}\left(x^{\prime}\right)-\mathcal{A} \circ f^{k+r}\left(y^{\prime}\right)\right| \leq \frac{C}{\lambda^{\alpha N^{\prime}}}
$$

Lemma (3.8) yields again

$$
\sum_{k=0}^{N^{\prime}}\left|\mathcal{A} \circ f^{k+r}\left(x^{\prime}\right)-\mathcal{A} \circ f^{k+r}\left(y^{\prime}\right)\right| \leq C \eta(x, y)
$$

On the other hand, $d\left(f\left(z_{1}\right), f\left(z_{2}\right)\right)$ is less than $C d\left(z_{1}, z_{2}\right)$ with $C=\|\| d f\|\|$. This implies that

$$
\sum_{k=N^{\prime}+1}^{N^{\prime}+p}\left|\mathcal{A} \circ f^{k+r}\left(x^{\prime}\right)-\mathcal{A} \circ f^{k+r}\left(y^{\prime}\right)\right| \leq C\left(d\left(f^{N^{\prime}+r}\left(x^{\prime}\right), f^{N^{\prime}+r}\left(y^{\prime}\right)\right)\right)^{\alpha},
$$

and finally, we obtain

$$
\left|\sum_{k=0}^{N} \mathcal{A} \circ f^{k+r}\left(x^{\prime}\right)-\mathcal{A} \circ f^{k+r}\left(y^{\prime}\right)\right| \leq C \eta(x, y)
$$

where $C$ is a universal constant.

- upper bound of second term: Just like the previous term, except we don't need to use Lemma (3.8).

- upper bound of third and fourth terms: This is a direct consequence of contraction along the stable leaves and the fact that $\mathcal{A}$ is Hölder continuous.

Remark. Using the contraction along stable leaves, we have $|\omega(x)| \leq \operatorname{Cdiam}(R)$ for all $x$ in $R_{1}$ and for all 1-cylinders containing $x$.

If $x$ is a point in $R_{n} \backslash \partial_{g} F, S_{n}(\mathcal{B})(x)$ will denote $\sum_{k=0}^{n-1} \mathcal{B}\left(g_{F}^{k}(x), C_{i}^{k}\right)$, where $C_{i}^{k}$ is the 1-cylinder containing $g_{F}^{k}(x)$. If it belongs to $\partial_{g} F$, then $S_{n}(\mathcal{B})(x)$ will denote the same expression in which we have just exchanged $g_{F}^{k}$ by the projection onto $F$ by $\pi_{F}$ of good iterates of $f$ (corresponding to the $k$ th return time of the $n$-cylinder). We have

Lemma 3.13. Let $n \in \mathbb{N}, x$ and $y$ be two points in $F,\left(x^{\prime}, y^{\prime}\right) \in \operatorname{Ant}_{n}(x) \times A_{n} t_{n}(y)$ such that $x^{\prime}$ and $y^{\prime}$ are in the same $n$-cylinder; then there exists a universal constant $C$ such that

$$
\left|S_{n}(\mathcal{B})\left(x^{\prime}\right)-S_{n}(\mathcal{B})\left(y^{\prime}\right)\right| \leq C \eta(x, y) .
$$

Proof. Pick $n \in \mathbb{N}$ and $x$ and $y$ two points in $F$. Since the interior of $\partial F$ is empty, we assume that neither $x$ nor $y$ are in $\partial F$. This guarantees that for any $x^{\prime} \in A n t_{n}(x)$ and $y^{\prime} \in \operatorname{Ant}_{n}(y)$ neither $x^{\prime}$ nor $y^{\prime}$ are in $\partial_{g} F$.

We can show by induction that

$$
S_{n}(\mathcal{B})\left(x^{\prime}\right)=\sum_{k=0}^{r^{n}\left(x^{\prime}\right)-1} \mathcal{A} \circ f^{k}\left(x^{\prime}\right)+\omega\left(g^{n-1}\left(x^{\prime}\right)\right),
$$


hence

$$
\begin{aligned}
\left|S_{n}(\mathcal{B})\left(x^{\prime}\right)-S_{n}(\mathcal{B})\left(y^{\prime}\right)\right| \leq & \sum_{k=0}^{r^{n}\left(x^{\prime}\right)-1}\left|\mathcal{A} \circ f^{k}\left(x^{\prime}\right)-\mathcal{A} \circ f^{k}\left(y^{\prime}\right)\right| \\
& +\left|\omega\left(g^{n-1}\left(x^{\prime}\right)\right)-\omega\left(g^{n-1}\left(y^{\prime}\right)\right)\right| .
\end{aligned}
$$

Lemma (3.12) shows that $\left|\omega\left(g^{n-1}\left(x^{\prime}\right)\right)-\omega\left(g^{n-1}\left(y^{\prime}\right)\right)\right| \leq C \eta(x, y)$, where $C$ is a universal constant. Thus the expanding property and the Hölder continuity of $\mathcal{A}$ and $\pi_{F}$ finally lead to

$$
\sum_{k=0}^{r^{n}\left(x^{\prime}\right)-1}\left|\mathcal{A} \circ f^{k}\left(x^{\prime}\right)-\mathcal{A} \circ f^{k}\left(y^{\prime}\right)\right| \leq C \eta(x, y) .
$$

If $x$ or $y$ is in $\partial F$, we use continuity.

\section{Existence of Gibbs measures for $\left(F, g_{F}\right)$}

4.1. The Perron-Frobenius operator. Let $S \in \mathbb{R}$; we define $\mathcal{B}_{S}$ by $\mathcal{B}_{S}\left(x, C_{i}\right) \stackrel{\text { def }}{=}$ $\mathcal{B}\left(x, C_{i}\right)-S r$, and a family of operators $\mathcal{L}_{S}$.

Definition 4.1. For $S \in \mathbb{R}$, we denote by $\mathcal{L}_{S}$ the operator defined by

$$
\begin{aligned}
\mathcal{L}_{S}: \phi \in C^{0}(F) \longmapsto \mathcal{L}_{S}(\phi): F & \longrightarrow \mathbb{R}, \\
x & \longmapsto \mathcal{L}_{S}(\phi)(x) \stackrel{\text { def }}{=} \sum_{y \in \operatorname{Ant}(x)} e^{\mathcal{B}_{S}\left(y, C_{y}\right)} \phi(y) .
\end{aligned}
$$

We claim that if $\mathcal{L}_{S}\left(\mathbb{1}_{F}\right)(x)<+\infty$ for some $x$, then $\mathcal{L}_{S^{\prime}}\left(\mathbb{1}_{F}\right)(y)<+\infty$ for all $y$ in $F$ and all $S^{\prime} \geq S$. This allow us to define Critical-Convergence-Value of these operators.

Definition 4.2. We will call Critical-Convergence-Value of $\mathcal{L}_{S}$, denoted by $S_{s}$, the infinimum in $\overline{\mathbb{R}}$ of the set of $S^{\prime}$ such that

$$
\forall S>S^{\prime} \forall x \in F \quad \mathcal{L}_{S}\left(\mathbb{1}_{F}\right)(x)<+\infty .
$$

Proposition 4.3. The Critical-Convergence-Value of $\mathcal{L}_{S}$ is less than or equal to the topological pressure of $(\Omega, f)$ for the potential $\mathcal{A}$.

Proof. Let us pick an $x$ in $F$; then $\mathcal{L}_{S}\left(\mathbb{1}_{F}\right)(x)=\sum_{n=1}^{+\infty}\left(\sum_{y \in A n t(n, x)} e^{\mathcal{B}\left(y, C_{y}\right)}\right) e^{-n S}$. It is sufficient to look for the convergence radius of this power series. If $y$ and $y^{\prime}$ are two points in $\operatorname{Ant}(n, x)$, then they must be $(\varepsilon, n)$-separated, because they are in the same local unstable manifold at time 0 and in the same local stable manifold at time $n$. This means that $\operatorname{Ant}(n, x)$ is an $(\varepsilon, n)$-separated set of points, which is not maximal, and

$$
\limsup _{n \rightarrow+\infty} \frac{1}{n} \log \left(\sum_{y \in \operatorname{Ant}(n, x)} e^{\mathcal{B}\left(y, C_{y}\right)}\right) \leq \mathcal{P}_{\mathcal{A}} .
$$

Cauchy's criterion implies that $S_{S} \leq \mathcal{P}_{\mathcal{A}}$.

Remark. We will see later that for $S=\mathcal{P}_{\mathcal{A}}$ the series converges. 
From now till the end, we will assume that $S>S_{s}$.

We will denote by $M_{S}$ the real number $\sup _{x \in F}\left|\mathcal{L}_{S}(\mathbb{1})(x)\right|$.

Proposition 4.4. $\mathcal{L}_{S}$ is a bounded operator from $\left(C^{0}(F),\|\|_{\infty}\right)$ to itself.

Proof. Pick $\varphi$ in $C^{0}(F)$; we have

$$
\begin{aligned}
\left|\mathcal{L}_{S}(\varphi)(x)-\mathcal{L}_{S}(\varphi)(y)\right| \leq & \sum_{k=1}^{+\infty} \sum_{\substack{x^{\prime} \in \operatorname{Ant}(k, x) \\
y^{\prime} \in \operatorname{Ant}(k, y)}}\left|e^{\mathcal{B}_{S}\left(x^{\prime}\right)} \varphi\left(x^{\prime}\right)-e^{\mathcal{B}_{S}\left(y^{\prime}\right)} \varphi\left(y^{\prime}\right)\right| \\
\leq & \sum_{\substack{k=1 \\
x^{\prime} \in \operatorname{Ant}(k, x) \\
y^{\prime} \in \operatorname{Ant}(k, y)}} e^{\mathcal{B}_{S}\left(x^{\prime}\right)}\left|\varphi\left(x^{\prime}\right)-\varphi\left(y^{\prime}\right)\right| \\
& +\|\varphi\|_{\infty} \sum_{k=1}^{+\infty} \sum_{\substack{x^{\prime} \in \operatorname{Ant}(k, x) \\
y^{\prime} \in \operatorname{Ant}(k, y)}}\left|e^{\mathcal{B}_{S}\left(x^{\prime}\right)}-e^{\mathcal{B}_{S}\left(y^{\prime}\right)}\right| .
\end{aligned}
$$

Using the fact that $\varphi$ is uniformly continuous on the compact set $F$, and using Lemma (3.13), we easily check that $\mathcal{L}_{S}(\varphi) \in C^{0}(F)$. Thus $\left\|\mathcal{L}_{S}(\varphi)\right\|_{\infty} \leq M_{S}\|\varphi\|_{\infty}$.

4.2. Definition of $\mu_{S} \cdot \mathcal{L}_{S}$ is a bounded operator from the space of continuous functions on a compact set to itself. It admits an adjoint operator, denoted by $\mathcal{L}_{S}^{*}$, acting on the set of probability measures (denoted by $\mathcal{M}_{F}$ ) and defined in the following way:

$$
\mathcal{L}_{S}^{*}(\mu) \cdot \stackrel{\text { def }}{=} \int \mathcal{L}_{S}(\varphi) d \mu
$$

Schauder-Tychonoff's theorem implies the existence of an eigenvalue for this adjoint operator.

Proposition 4.5. There exist a probability measure $\mu_{S}$ and a real number $\lambda_{S} \in \mathbb{R}_{+}^{*}$ such that

$$
\mathcal{L}_{S}^{*}\left(\mu_{S}\right)=\lambda_{S} \mu_{S}
$$

Definition 4.6. Every element of $\mathcal{M}_{F}$ which is a fixed point of $\widetilde{\mathcal{L}}_{S}^{*} \stackrel{\text { def }}{=} \frac{1}{\lambda_{S}} \mathcal{L}_{S}^{*}$ will be called the Gibbs measure associated to the potential $\mathcal{B}_{S}$.

Proposition 4.7. For any Gibbs-measure $\mu_{S}$, the set $H$ of points in $F$ that return only finitely often in $R$ by the map $f$ has zero measure.

Proof. Let $n \in \mathbb{N}$, and let $H_{1}^{n-1}$ denote the set of points that return only $n-1$ times in $R$ by the map $f$, and $K_{n-1}$ be a compact set in $H_{1}^{n-1}$. Let $\delta>0$; there is an integer $N$ such that for all $x$ in $F$

$$
\sum_{k=N}^{+\infty} \sum_{\substack{y \in A n t_{n}(x) \\ r^{n}\left(C_{n}(y)\right)=k}} e^{S_{n}\left(\mathcal{B}_{S}\right)\left(y, C_{n}(y)\right)}<\delta \lambda_{S}^{n}
$$

where $C_{n}(y)$ denotes an $n$-cylinder containing $y$, and $r^{n}\left(C_{n}(y)\right)$ denotes the $n$-th return time. $F_{\delta}^{n}=\bigcup_{r^{n}}\left(C_{n}(y)\right) \leq N=C_{n}(y)$ is compact. By Urysohn's Lemma (see [14]), there exists some continuous function $\varphi_{\delta, n}$, such that $\varphi_{\delta, n}(x)=1$, for all 
$x \in K_{n-1}, 0 \leq \varphi_{\delta, n} \leq 1$, and $\varphi_{\delta, n}(x)=0$, for all $x \in F_{\delta}^{n}$. Then

$$
\mu_{S}\left(K_{n}\right) \leq \frac{1}{\lambda_{S}^{n}} \int \mathcal{L}_{S}^{n}\left(\varphi_{\delta, n}\right) d \mu_{S} \leq \delta
$$

hence $\mu_{S}(H)=0$.

4.3. Hypotheses of Ionescu-Tulcea and Marinescu's theorem holds. We want to check that Ionescu-Tulcea and Marinescu's theorem's hypotheses (see C] in appendix) hold for the operator $\widetilde{\mathcal{L}}_{S}$. The small space will be $\left(C_{\eta}^{0}(F),\|\|_{\eta}\right)$ and the large one $\left(C^{0}(F),\|\|_{\infty}\right)$. Complex valued functions are considered here as $\varphi=\varphi_{r}+i \varphi_{i}$.

(i) holds: Let $\left(\varphi_{n}\right)_{n \in \mathbb{N}}$ be a sequence of functions in $C_{\eta}^{0}(F)$, converging to $\varphi$ in $\left(C^{0}(F),\|\|_{\infty}\right)$. Let $C$ be a constant such that, for all $n,\left\|\varphi_{n}\right\|_{\eta} \leq C$. Pick $\delta>0$ and $N$ such that, for all $n \geq N$,

$$
\left\|\varphi_{n}-\varphi\right\|_{\infty} \leq \delta
$$

If $x$ and $y$ are two points in $F$ and $n$ is an integer greater than $N$, then

$$
\begin{aligned}
|\varphi(x)-\varphi(y)| & \leq 2 \delta+\left|\varphi_{n}(x)-\varphi_{n}(y)\right| \\
& \leq 2 \delta+\left(C-\left\|\varphi_{n}\right\|_{\infty}\right) \eta(x, y) \\
& \leq 2 \delta+\left(C-\|\varphi\|_{\infty}+\delta\right) \eta(x, y)
\end{aligned}
$$

which shows that $\varphi \in C_{\eta}^{0}(F)$ and $\|\varphi\|_{\eta} \leq C$.

(ii) and (iv) hold: Pick $\varphi \in C_{\eta}^{0}(F)$ and denote by $C_{\varphi}$ the real number

$$
\sup _{x \neq y} \frac{|\varphi(x)-\varphi(y)|}{\eta(x, y)} \text {. }
$$

Let $x$ and $y$ be in $F$, and $n$ an integer; we obtain by Lemma (

$$
\begin{aligned}
\left|\widetilde{\mathcal{L}_{S}}{ }^{n}(\varphi)(x)-\widetilde{\mathcal{L}_{S}}{ }^{n}(\varphi)(y)\right| \leq & \frac{C_{\varphi}}{\lambda_{S}^{n}} \sum_{\substack{x^{\prime} \in \operatorname{Ant}_{n}(x) \\
y^{\prime} \in A n t_{n}(y)}} e^{S_{n}\left(\mathcal{B}_{S}\right)\left(x^{\prime}, C_{n}\left(x^{\prime}, y^{\prime}\right)\right)} \eta\left(x^{\prime}, y^{\prime}\right) \\
& +\frac{1}{\lambda_{S}^{n}}\|\varphi\|_{\infty} \sum_{\substack{x^{\prime} \in A n t_{n}(x) \\
y^{\prime} \in A n t_{n}(y)}} e^{S_{n}\left(\mathcal{B}_{S}\right)\left(y^{\prime}, C_{n}\left(x^{\prime}, y^{\prime}\right)\right)}\left|e^{C_{\mathcal{B}} \eta(x, y)}-1\right| .
\end{aligned}
$$

Lemma 3.8 proves that $N\left(x^{\prime}, y^{\prime}\right) \approx N(x, y)+\frac{r^{n}\left(C_{n}\left(x^{\prime}, y^{\prime}\right)\right)}{2}$.

So there exists a universal constant $C$ such that

$$
\begin{aligned}
\left|\widetilde{\mathcal{L}}_{S}^{n}(\varphi)(x)-{\widetilde{\mathcal{L}_{S}}}^{n}(\varphi)(y)\right| \leq & C_{\varphi} \eta(x, y)\left(\frac{C}{\lambda_{S}^{n}} \sum_{x^{\prime} \in A n t_{n}(x)} \frac{e^{S_{n}\left(\mathcal{B}_{S}\right)\left(x^{\prime}\right)}}{\lambda^{\frac{\alpha \gamma}{2} r^{n}\left(x^{\prime}\right)}}\right) \\
& +\eta(x, y)\|\varphi\|_{\infty}\left(\frac{C}{\lambda_{S}^{n}} \sum_{y^{\prime} \in A n t_{n}(y)} e^{S_{n}\left(\mathcal{B}_{S}\right)\left(y^{\prime}\right)}\right) .
\end{aligned}
$$

For $n=1$ we get from (2) that $\widetilde{\mathcal{L}_{S}}(\varphi) \in C_{\eta}^{0}(F)$ and that $\widetilde{\mathcal{L}_{S}}$ is bounded for \|\|$_{\eta}$.

$S_{n}\left(\mathcal{B}_{S}\right)$ is continuous on each $n$-cylinder which is a compact set. It is bounded from above and we have by Lemma (3.13) that

$$
\lambda_{S}^{n}=\int \mathcal{L}_{S} \mathbb{1}_{F} d \mu_{S} \geq \sum_{C_{n} n \text {-cylinder }} e^{-C} \exp \left(\sup _{C_{n}} S_{n}\left(\mathcal{B}_{S}\right)\right) .
$$


This gives

$$
\begin{aligned}
& \frac{C}{\lambda_{S}^{n}} \sum_{x^{\prime} \in A_{n t_{n}}(x)} \frac{\exp \left(S_{n}\left(\mathcal{B}_{S}\right)\left(x^{\prime}, C_{n}\left(x^{\prime}\right)\right)\right)}{\lambda^{\frac{\alpha \gamma}{2}} r^{n}\left(C_{n}\left(x^{\prime}\right)\right)} \\
& \leq \frac{\sum_{x^{\prime} \in \operatorname{Ant}_{n}(x)} C e^{C} \frac{\exp \left(S_{n}\left(\mathcal{B}_{S}\right)\left(x^{\prime}, C_{n}\left(x^{\prime}\right)\right)\right)}{\lambda^{\frac{\alpha \gamma}{2}} r^{n}\left(x^{\prime}, C_{n}\left(x^{\prime}\right)\right)}}{\sum_{x^{\prime} \in \operatorname{Ant}_{n}(x)} \exp \left(\sup _{C_{n}\left(x^{\prime}\right)} S_{n}\left(\mathcal{B}_{S}\right)\right)} .
\end{aligned}
$$

As $r^{n}\left(x^{\prime}, C_{n}\left(x^{\prime}\right)\right) \geq n$ for all $n$ and all $x^{\prime} \in A n t_{n}(x)$, we pick $n_{0}$ such that $\lambda^{\frac{\alpha \gamma}{2} n_{0}} \geq$ $2 C e^{C}$. Then (3) yields

$$
\left\|\widetilde{\mathcal{L}}_{S}^{n_{0}}(\varphi)\right\|_{\eta} \leq \frac{1}{2}\|\varphi\|_{\eta}+b\|\varphi\|_{\infty}
$$

for some $0 \leq b<+\infty$.

(iii) and (v) hold: Using Lemmas (3.12) and (3.13), we check that $\left\|\widetilde{\mathcal{L}}_{S}^{n}\left(\mathbb{1}_{F}\right)\right\|_{\infty} \leq$ $e^{C}<\infty$. Moreover, if $\varphi \in C_{\eta}^{0}(F)$ and $\|\varphi\|_{\infty} \leq 1$, then, for all integers $n$, we have $\left\|\widetilde{\mathcal{L}}_{S}^{n}(\varphi)\right\|_{\infty} \leq\left\|\widetilde{\mathcal{L}}_{S}^{n}\left(\mathbb{1}_{F}\right)\right\|_{\infty}$. For $(v)$, Ascoli's theorem is needed.

\subsection{Quasi-Gibbs measures.}

4.4.1. $\lambda_{S}$ is an eigenvalue of $\mathcal{L}_{S}$. Using Ionescu-Tulcea and Marinescu's theorem we get the following result.

Proposition 4.8. There exists $h \in C_{\eta}^{0}(F)$, a strictly positive function, with $\mu_{S^{-}}$ integral equal to 1 such that $\mathcal{L}_{S}(h)=\lambda_{S} h$.

Proof. Lemma (C.3) shows that there exists $h \in C_{\eta}^{0}(F)$ such that

$$
\lim _{n \rightarrow+\infty}\left\|\frac{1}{n} \sum_{k=0}^{n-1} \widetilde{\mathcal{L}}_{S}^{k}\left(\mathbb{1}_{F}\right)-h\right\|_{\infty}=0 .
$$

Obviously $\widetilde{\mathcal{L}}_{S}(h)=h$. Moreover, $\mathcal{L}_{S}$ is a positive operator, because $\mathcal{B}_{S}$ is a realvalued function; this implies that $h$ is positive. Also, Lebesgue's dominated convergence theorem shows that $\int h d \mu_{S}=1$, and the mixing property proves that $h$ cannot vanish.

4.4.2. Property of Gibbs measures. As we have seen in previous sections, problems on $F$ come from points of $H$ or $\partial_{g} F$. Unfortunatly we have to keep these sets to find good measures among the dual set of the continuous functions on the compact set $F \supset H \cup \partial_{g} F$. We have already seen that $\mu_{S}(H)=0$ for any Gibbs measure $\mu_{S}$. We see now another important result

Proposition 4.9. For any Gibbs measure $\mu_{S}, \partial F$ and $A n t_{n}(\partial F)$ have zero measure for all $n \in \mathbb{N}$.

Proof. Pick $n$ in $\mathbb{N}^{*}$. Set $A n t_{n}(\partial F) \cap \operatorname{int}(F) \stackrel{\text { def }}{=} D_{n}^{\circ}$. Since $D_{n}^{\circ} \subset \operatorname{int}(F)$, no point can be in more than $n$ distinct $\operatorname{Ant}_{k}\left(D_{n}^{\circ}\right)\left(k \in \mathbb{N}^{*}\right)$. Use Urysohn's lemma and Lebesgue's convergence theorem to check that

$$
\mu_{S}\left(\operatorname{Ant}_{k}\left(D_{n}^{\circ}\right)\right)=\frac{1}{\lambda_{S}^{k}} \int \mathbb{1}_{D_{n}^{\circ}} \cdot \widetilde{\mathcal{L}}_{S}^{k}\left(\mathbb{1}_{F}\right) d \mu_{S} .
$$

Then set $D_{n}^{\infty} \stackrel{\text { def }}{=} \bigcup_{k \in \mathbb{N}^{*}} A n t_{k}\left(D_{n}^{\circ}\right)$; Lemma (C.3) yields again that $\mu_{S}\left(D_{n}^{\circ}\right)=0$. 
Pick an $n$-cylinder $C_{n}$ included in $\operatorname{int}(F)$; check that

$$
0=\mu_{S}\left(\partial C_{n}\right)=\int \psi(y) \mathbb{1}_{\partial F}(y) d \mu_{S}(y)
$$

where $\psi$ is bounded from below on $\partial F$. Then $\mu_{S}(\partial F)=0$.

4.4.3. Invariant quasi-Gibbs measure. From now on, we can assume that, modulo any Gibbs measure, cylinders constitute an increasing family of partitions. It allows us to consider that $\mathcal{B}$ and $\omega$ are well defined functions on a set of full measure. We claim that we can extend $\mathcal{L}_{S}$ on $L^{1}\left(\mu_{S}\right)$ for any Gibbs measure.

Proposition 4.10. For any Gibbs measure $\mu_{S}, \mathcal{L}_{S}$ can be extended to a contracting bounded operator from $L^{1}\left(\mu_{S}\right)$ to itself.

Remark. For all $\phi$ in $L^{1}\left(\mu_{S}\right)$, we have $\int \widetilde{\mathcal{L}}_{S}(\phi) d \mu_{S}=\int \phi d \mu_{S}$.

Lemma 4.11. Let $\varphi \in L^{1}\left(\mu_{S}\right)$; then $\widetilde{\mathcal{L}}_{S}(\varphi)=\varphi \mu_{S}$-a.e. if and only if the measure $m_{S}$ defined by $d m_{S}=\varphi d \mu_{S}$ is $g_{F}$-invariant.

Proof. This is a very classical computation because $H$ and $\partial_{g} F$ have zero measure.

A direct application of Lemma (4.11) is the existence of quasi-Gibbs invariant measure.

Definition 4.12. We call quasi-Gibbs measure any measure $m_{S}$ defined by $d m_{S} \stackrel{\text { def }}{=}$ $h d \mu_{S}$ where $\mu_{S}$ is a Gibbs measure.

It is called quasi-Gibbs invariant measure iff it is a $g_{F}$-invariant quasi-Gibbs measure.

\section{Uniqueness of the GibBs meAsures FOR $\left(F, g_{F}\right)$}

5.1. Some technical lemmas. In this section we briefly recall a well-known Martingale theorem.

For any integer $k, \mathcal{Q}_{k}$ will denote the $\sigma$-algebra generated by the $k$-cylinders family, and $\mathcal{E}$ will denote the trivial $\sigma$-algebra, meaning the $\sigma$-algebra whose atoms are points. Obviously, $\left(\mathcal{Q}_{k}\right)_{k \in \mathbb{N}^{*}}$ is an increasing sequence with respect to the measure $\mu_{S}$, which converges to $\mathcal{E}$. As the $k$-cylinders are a partition with respect to $\mu_{S}$, the conditional expectation of any measurable function $\phi$ satisfies, for $\mu_{S^{-}}$ almost every point $x$,

$$
\phi_{k} \stackrel{\text { def }}{=} \mathbb{E}\left[\phi \mid \mathcal{Q}_{k}\right](x)=\frac{1}{\mu_{S}\left(C_{k}(x)\right)} \int_{C_{k}(x)} \phi d \mu_{S}
$$

where $C_{k} \in \mathcal{Q}_{k}$ is the $k$-cylinder containing $x$. Then we have

Proposition 5.1 (Martingale theorem). If $\phi \in L^{1}\left(\mu_{S}\right)$, then $\phi_{k} \longrightarrow \phi \mu_{S}$-almost everywhere when $k \rightarrow+\infty$.

This gives the following corollary.

Lemma 5.2. For all Borel sets $A$ in $F$, for $\mu_{S}$-almost every $x$

$$
\lim _{k \rightarrow+\infty} \frac{\mu_{S}\left(A \cap C_{k}(x)\right)}{\mu_{S}\left(C_{k}(x)\right)}=\mathbb{1}_{A}(x)
$$

where $C_{k}(x)$ is the $k$-cylinder containing $x$. 
Proof. Just use Proposition (5.1) with $\phi=\mathbb{1}_{A}$.

Lemma 5.3. Any Gibbs measure $\mu_{S}$ is continuous.

Proof. Check that any atom $x$ must be a periodic point, and that the conformal condition implies every $y \in A n t(x)$ is also an atom. Then $A n t(x)$ must be in $x$ 's orbit.

5.2. Uniqueness of $\mu_{S}$ and $m_{S}$. In this subsection, we will see that there exist only one Gibbs measure and one invariant quasi-Gibbs measure. This is the consequence of the fact that any Gibbs measure is a conformal measure (see Appendix B).

Definition 5.4. For $n \in \mathbb{N}$ we denote by $\mathcal{T}_{n}$ the $\sigma$-algebra of Borel sets such that if $E \in \mathcal{T}_{n}$ and $x \in E$, then

$$
\operatorname{Ant}_{n}\left(g_{F}^{n}(x)\right) \subset E .
$$

If $x$ is in $A n t_{n}(y)$, we will call $n$-brother of $x$ any element of $A n t_{n}(y)$.

We have the following property, which specifies how any Gibbs measure is well distributed.

Proposition 5.5. Any Gibbs measure $\mu_{S}$ is exact, meaning that $\bigcap_{n \in \mathbb{N}} \mathcal{T}_{n}=\{\emptyset, F\}$ for the measure $\mu_{S}$.

Proof. Let $E$ be a Borel set in $\mathcal{T}_{\infty} \stackrel{\text { def }}{=} \bigcap_{n \in \mathbb{N}} \mathcal{T}_{n}$. We can assume that $E \cap$ $\left(H \cup \partial_{g} F\right)=\emptyset$. If $\mu_{S}(E)>0$, then using Lemma (5.2) we find a point $x$ in $E$ such that $\lim _{k \rightarrow+\infty} \frac{\mu_{S}\left(E \cap C_{k}(x)\right)}{\mu_{S}\left(C_{k}(x)\right)}=\mathbb{1}_{E}(x)=1$. Set $\delta>0$; there exists $k_{1}$ such that for, all $k \geq k_{1}$, we have $\mu_{S}\left(E \cap C_{k}(x)\right) \geq(1-\delta) \mu_{S}\left(C_{k}(x)\right)$.

$E$ is a Borel set of $\mathcal{T}_{k_{1}}$ and by Lemma (3.13) we have

$$
\frac{\mu_{S}\left(E \cap C_{k_{1}}(y)\right)}{\mu_{S}\left(C_{k_{1}}(y)\right)} \geq(1-\delta) e^{-4 C}
$$

for all $y k_{1}$-brothers of $x$. Summing this inequality over all the $k_{1}$-brothers of $x$ we get

$$
\mu_{S}(E)=\sum_{y \in \text { Ant }_{k_{1}}\left(g_{F}^{k_{1}}(x)\right)} \mu_{S}\left(E \cap C_{k_{1}}(y)\right) \geq(1-\delta) e^{-4 C} .
$$

By Lemma 5.3 $\mu_{S}$ is non-atomic and we must have $\mu_{S}(E)=1$.

This property of any Gibbs measure yields a property for any correspondent invariant quasi-Gibbs measure.

Proposition 5.6. Any invariant quasi-Gibbs measure $m_{S}$ is mixing, thus ergodic.

Proof. Let $\phi$ and $\psi$ be two continuous and zero-integral functions. We will prove that

$$
\lim _{n \rightarrow+\infty} \int \phi \cdot \psi \circ g_{F}^{n} d m_{S}=0
$$

$\int \phi . \psi \circ g_{F}^{n} d m_{S}=\int \mathbb{E}\left[\phi . \psi \circ g_{F}^{n} \mid \mathcal{T}_{n}\right] d m_{S}$, which is equal to $\int \mathbb{E}\left[\phi \mid \mathcal{T}_{n}\right] . \psi \circ g_{F}^{n} d m_{S}$ by the definition of conditional expectation. $m_{S}$ and $\mu_{S}$ are equivalent and the $\sigma$-algebra $\mathcal{T}_{n}$ decreases to the trivial $\sigma$-algebra. Moreover

$$
\left\|\mathbb{E}\left[\phi \mid \mathcal{T}_{n}\right] . \psi \circ g_{F}^{n}\right\|_{\infty} \leq\|\psi\|_{\infty}\|\phi\|_{\infty} .
$$


Lebesgue's dominated convergence theorem yields

$$
\lim _{n \rightarrow+\infty} \int \phi . \psi \circ g_{F}^{n} d m_{S}=0 .
$$

Ergodicity can be seen as an extremal property in the convex compact set of invariant probability measures. We will use this point of view to prove uniqueness of the Gibbs measure and the invariant quasi-Gibbs measure.

Proposition 5.7. The system $\left(F, g_{F}\right)$ admits a unique Gibbs measure associated to the potential $\mathcal{B}_{S}$ and a unique invariant quasi-Gibbs measure.

Proof. Uniqueness of the Gibbs measure is a consequence of the mixing property of the map (Ruelle's Perron-Frobenius theorem): The operator $\mathcal{L}_{S}$ has a strictly positive real eigenvalue of greatest module, which is $\lambda_{S}$. Hence, any Gibbs measure is associated to this eigenvalue $\lambda_{S}$ for the operator $\mathcal{L}_{S}^{*}$. If $\widetilde{\mu}_{S}$ is a Gibbs measure, we set for any $t \in[0,1] \mu_{S}^{t} \stackrel{\text { def }}{=} t \mu_{S}+(1-t) \widetilde{\mu}_{S}$, and $m_{S}^{t}$ the measure defined by $d m_{S}^{t}=h d \mu_{S}^{t}$. Since $m_{S}^{t}$ is ergodic for any $t, \widetilde{\mu}_{S}$ must be equal to $\mu_{S}$.

We show in the same way that the eigenvector $h$ of $\mathcal{L}_{S}$ associated to $\lambda_{S}$ in $L^{1}\left(\mu_{S}\right)$ must be unique, which proves the uniqueness of the invariant quasi-Gibbs measure.

5.3. $m_{S}$ 's property. For the dynamical system $\left(F, g_{F}\right)$, we will see that there are Equilibrium States just as for any Axiom-A. We first define

Definition 5.8. Set $\mathcal{M}_{F}^{\prime}$ to be the set of $g_{F}$-invariant ergodic probability measures, such that $H \cup \partial F$ has zero measure. If $m$ is in $\mathcal{M}_{F}^{\prime}$, we denote by $\mathcal{P}_{m}\left(\mathcal{B}_{S}\right)$ the real number $h_{m}\left(g_{F}\right)+\int \mathcal{B}_{S} d m$, and we call it the metric pressure of the measure $m$ for the potential $\mathcal{B}_{S}$. We will call pressure of the system for the potentiel $\mathcal{B}_{S}$, denoted by $\mathcal{P}\left(\mathcal{B}_{S}\right)$, the element of $\overline{\mathbb{R}}$ defined by

$$
\mathcal{P}\left(\mathcal{B}_{S}\right)=\sup _{m \in \mathcal{M}_{F}^{\prime}} \mathcal{P}_{m}\left(\mathcal{B}_{S}\right)
$$

Any measure which realizes this maximum will be called an Equilibrium State for $\mathcal{B}_{S}$.

We have the following result.

Proposition 5.9. For every Hölder-continuous function $\mathcal{A}$ defined on $\Omega$, for every $S>S_{S}$ there exists a unique Equilibrium State for $\mathcal{B}_{S}$, which is the unique $g_{F^{-}}$ invariant quasi-Gibbs measure associated to $\mathcal{B}_{S}$. Moreover, the pressure of the system is $\log \lambda_{S}$.

Proof. Using ideas given in [15], [9] and [3], we check that we have just to prove the following lemma.

Lemma 5.10. There exists a universal constant $C$ such that, for all $n \in \mathbb{N}$, for all $n$-cylinders $C_{n}$, and for all $x$ in $C_{n}$ we have

$$
\frac{1}{C} \leq \frac{m_{S}\left(C_{n}\right)}{\frac{1}{\lambda_{S}^{n}} e^{S_{n}\left(\mathcal{B}_{S}\right)(x)}} \leq C .
$$


Proof. Let $n$ be an integer. Pick $C_{n}$ an $n$-cylinder and let $x$ be in $C_{n} . C_{n}$ is a compact set in $F$. As $m_{S}(\partial F)=0$ we have

$$
m_{S}\left(C_{n}\right)=m_{S}\left(\operatorname{int}\left(C_{n}\right)\right)=\sup _{\varphi \prec \operatorname{int}\left(C_{n}\right)} \int \varphi d m_{S}
$$

where $\varphi \prec \operatorname{int}\left(C_{n}\right)$ means that $\varphi$ is a positive continuous function, with support in $\operatorname{int}\left(C_{n}\right)$, and bounded from above by 1 . Pick such a function $\varphi$. Then we have

$$
\begin{aligned}
\int \varphi d m_{S} & \stackrel{\text { def }}{=} \int \varphi h d \mu_{S} \\
& =\frac{1}{\lambda_{S}^{n}} \int \sum_{y \in A_{n t_{n}(x)}} e^{S_{n}\left(\mathcal{B}_{S}\right)(y)} \varphi(y) \cdot h(y) d \mu_{S}(x) \\
& =\frac{1}{\lambda_{S}^{n}} \int \mathbb{1}_{C_{n}}(y) e^{S_{n}\left(\mathcal{B}_{S}\right)(y)} \varphi(y) \cdot h(y) d \mu_{S}(x) .
\end{aligned}
$$

There exists a universal constant $C$ such that

$$
\frac{1}{C} \leq h \leq C .
$$

Moreover, the variation of $\mathcal{B}_{S}$ on each cylinder is uniformly bounded by a universal constant. Equality (4) yields

$$
\frac{1}{C} \times\left(\frac{1}{\lambda_{S}^{n}} e^{S_{n}\left(\mathcal{B}_{S}\right)(x)}\right) \leq \int \varphi d m_{S} \leq C \times\left(\frac{1}{\lambda_{S}^{n}} e^{S_{n}\left(\mathcal{B}_{S}\right)(x)}\right),
$$

which proves Lemma 5.10 .

\section{Extensions}

6.1. Extensions of $\left(F, g_{F}, m_{\mathcal{P}_{S}}\right)$. In this subsection, we will prove that there exists a measure $\nu_{S}$ such that the system $\left(\Omega, f, \nu_{S}\right)$ can be seen as an extension of the system $\left(F, g_{F}, m_{S}\right)$.

6.1.1. Natural extension of $\left(F, g_{F}, m_{S}\right)$. We defined the system $\left(F, g_{F}\right)$ as a factor of the system $(R, g)$. We first want to check we can find a measure $\widehat{m}_{S}$ on the Borel set $\sigma$-algebra of $R$ which projects itself onto $m_{S}$ by $\pi_{F}$. In this aim, we have to define a new map $\widetilde{g}$ to solve problems of boundaries.

For $x$ in $R$, we know that $\pi_{F}(x) \notin \partial^{u} R$, and we set $\widetilde{g}(x) \stackrel{\text { def }}{=} f^{r\left(\pi_{F}(x)\right)}(x)$. Then we define a family of Borel sets in $R$ in the following way.

Definition 6.1. We call block of $R$ every Borel set $A$ such that either $R$ is empty, or if $x \in A$, then $W^{s}(x, R) \subset A$.

We define a map $\widehat{m}_{S}$ by

$\widehat{m}_{S}(A)= \begin{cases}m_{S}\left(\pi_{F}(A)\right) & \text { if } A \text { is a block, } \\ m_{S}\left(\pi_{F}\left(\widetilde{g}^{-n}(A)\right)\right) & \text { if } n \text { is the lowest integer s.t. } \widetilde{g}^{-n}(A) \text { is a block. }\end{cases}$

If we set $\mathcal{T} \stackrel{\text { def }}{=}\left\{\widetilde{g}^{n}(B), n \in \mathbb{N}, B\right.$ block $\}$, then we check that $\mathcal{T}$ is an algebra, containing the closed sets. The property of sequential continuity holds, meaning that, for every decreasing sequence of elements in $\mathcal{T},\left(A_{n}\right)_{n \in \mathbb{N}}$ such that $\bigcap_{n \in \mathbb{N}} A_{n}=$ $\emptyset, \lim _{n \rightarrow+\infty} \widehat{m}_{S}\left(A_{n}\right)=0$. Using Carathéodory's theorem, we have the following result.

Proposition 6.2. $\widehat{m}_{S}$ is a $\widetilde{g}$-invariant Borel probability measure. 
By construction of $\widehat{m}_{S}$, for each Borel set of $F, B$, we have $\widehat{m}_{S}\left(\pi_{F}^{-1}(B)\right)=m_{S}(B)$ and we claim this implies that $\left(R, \widetilde{g}, \widehat{m}_{S}\right)$ is the natural extension to $\left(F, g_{F}, m_{S}\right)$. Moreover it's ergodic, and the Markov property yields that $\partial^{u} R$ is $\widetilde{g}$-invariant. Then we claim it must have zero measure. Hence we deduce the following corollary.

Corollary 6.3. The maps $\widetilde{g}$ and $g$ are $\widehat{m}_{S}$-almost everywhere equal. Thus $\left(R, g, \widehat{m}_{S}\right)$ is the natural extension to $\left(F, g_{F}, m_{S}\right)$, and $\widehat{m}_{S}$ is $g$-invariant and ergodic.

The measure $\widehat{m}_{S}$ satisfies also a variational principle.

Definition 6.4. Set $\widehat{\mathcal{B}}_{S}$ to be the function defined by

$$
\widehat{\mathcal{B}}_{S}(x) \stackrel{\text { def }}{=} \sum_{k=0}^{r(x)-1} \mathcal{A} \circ f^{k}(x)-r(x) S
$$

for every $x$ in $R_{1}$. We denote by $\mathcal{M}_{R}^{\prime}$ the set of $g$-invariant probability measures such that $R \backslash\left(R_{\infty} \cup \partial R\right)$ has zero measure. For $\nu \in \mathcal{M}_{R}^{\prime}$, we set $\mathcal{P}_{\nu}\left(\widehat{\mathcal{B}}_{S}\right) \stackrel{\text { def }}{=}$ $h_{\nu}(g)+\int \widehat{\mathcal{B}}_{S} d \nu$ and we call it the pressure of $\nu$ for the potential $\widehat{\mathcal{B}}_{S}$. Then we set

$$
\mathcal{P}\left(\widehat{\mathcal{B}}_{S}\right) \stackrel{\text { def }}{=} \sup _{\nu \in \mathcal{M}_{R}^{\prime}} \mathcal{P}_{\nu}\left(\widehat{\mathcal{B}}_{S}\right)
$$

and call it the pressure of the system for the potential $\widehat{\mathcal{B}}_{S}$. Any measure which realizes this maximum is called Equilibrium State.

We have the following result.

Proposition 6.5. The system $(R, g)$ has a unique Equilibrium State associated to $\widehat{\mathcal{B}}_{S}$, which is $\widehat{m}_{S}$.

Proof. It is sufficient to check that $\mathcal{B}_{S} \circ \pi_{F}$ and $\widehat{\mathcal{B}}_{S}$ are cohomologous for the map $g$. Then we use the properties of the natural extension.

As a direct corollary we have the following.

Corollary 6.6. $\widehat{m}_{S}$ is independent of the choice of the leaf $F$.

6.1.2. Extension of $\left(R, g, \widehat{m}_{S}\right)$. We want to see the system $\left(R, g, \widehat{m}_{S}\right)$ as an induced system $\left(\Omega, f, \nu_{S}\right)$. Proposition (B.2) tells us we have just to prove the following result.

Lemma 6.7. For each $S>S_{S}$, we have $\int r d \widehat{m}_{s}<+\infty$.

Proof. By definition of $\widehat{m}_{S}$ it is sufficient to prove that $\int r d m_{s}<+\infty$. We have $\int r d \mu_{S}=\frac{1}{\lambda_{S}} \int \mathcal{L}_{S}(r) d \mu_{S}$ with $\mathcal{L}_{S}(r)(x)=\sum_{k=1}^{+\infty}\left(\sum_{y \in A n t(k, x)} e^{\mathcal{B}_{S}(y)}\right) k e^{-k S}$ for any $x$ in $F \backslash\left(H_{1} \cup \partial_{g} F\right)$. Pick $x_{0}$ in $F \backslash\left(H_{1} \cup \partial_{g} F\right)$. Then $\mathcal{L}_{S}(r)\left(x_{0}\right)$ can be seen as the derivative series of the power series $\mathcal{L}_{S}\left(\mathbb{1}_{F}\right)\left(x_{0}\right)$ which converges uniformly because $S_{S}<S$. Lemma (3.13) yields $\int r d m_{S}<+\infty$.

Hence we have,

Proposition 6.8. For all $S>S_{S}$, there exists an invariant ergodic Borel probability measure $\nu_{S}$ such that $\left(\Omega, f, \nu_{S}\right)$ induces $\left(R, g, \widehat{m}_{S}\right)$. Thus, the pressure of $\nu_{S}$ associated to $\mathcal{A}$ satisfies

$$
\mathcal{P}_{\nu_{S}}(f, \mathcal{A})=S+\nu_{S}(R) \log \lambda_{S} .
$$


Proof. The existence of $\nu_{S}$ is a direct consequence of the previous lemma. Moreover, $\log \lambda_{S} \stackrel{\text { def }}{=} h_{\widehat{m}_{S}}(g)+\int \mathcal{B}_{S} d \widehat{m}_{S}=\frac{1}{\nu_{S}(R)}\left[h_{\nu_{S}}(f)+\int \mathcal{A} d \nu_{S}-S\right]$.

Remark. In particular we have $\lambda_{S} \leq 1, \forall S>S_{S}$.

6.2. The case $S=\mathcal{P}_{\mathcal{A}}$.

6.2.1. Existence of $\mu_{\mathcal{P}_{\mathcal{A}}}$. By Proposition (4.3), we have $S_{S} \leq \mathcal{P}_{\mathcal{A}}$, but we don't know if $S_{S}<\mathcal{P}_{\mathcal{A}}$ or not. However, we know that a sufficient condition for the existence of $\mu_{S}$ is that the series $\mathcal{L}_{S}\left(\mathbb{1}_{F}\right)(x)$ converges for one point $x$ in $F$. We will prove that this fact holds for $S=\mathcal{P}_{\mathcal{A}}$, and we will deduce existence and uniqueness of the Equilibrium State for the system associated to the potential $\mathcal{B}_{\mathcal{P}_{\mathcal{A}}}$. To make the reading easier, we will exchange the subscript $\mathcal{P}_{\mathcal{A}}$ for $\mathcal{A}$.

Proposition 6.9. There exists a universal constant $C$ such that for all $x$ in $F \backslash \partial F$ we have

$$
\sum_{y \in \operatorname{Ant}(x)} e^{\mathcal{B}_{\mathcal{A}}(y)} \leq C
$$

Proof. Pick an $x$ in $F \backslash \partial F$. Pick $S>\mathcal{P}_{\mathcal{A}}$. There exists a universal constant such that $\mathcal{L}_{S}\left(\mathbb{1}_{F}\right)(x) \leq e^{C} \mathcal{L}_{S}\left(\mathbb{1}_{F}\right)(y)$, which implies $\mathcal{L}_{S}\left(\mathbb{1}_{F}\right)(x) \leq e^{C} \lambda_{S}$. Hence $S \mapsto \mathcal{L}_{S}\left(\mathbb{1}_{F}\right)(x)$ is decreasing positive and bounded from above on $\left(\mathcal{P}_{\mathcal{A}},+\infty\right)$ and admits a limit when $S$ tends to $\mathcal{P}_{\mathcal{A}}$. Thus

$$
\mathcal{L}_{\mathcal{A}}\left(\mathbb{1}_{F}\right)(x) \leq e^{C} .
$$

This proposition proves that the previous results also hold for $S=\mathcal{P}_{\mathcal{A}}$. We have in particular the next result.

Proposition 6.10. The dynamical system $\left(F, g_{F}\right)$ admits a unique Equilibrium State associated with $\mathcal{B}_{\mathcal{A}}$, which is the unique invariant quasi-Gibbs measure $m_{\mathcal{A}}$. The pressure of the system is $\log \lambda_{\mathcal{A}}$.

Again the natural extension of the system $\left(F, g_{F}, m_{\mathcal{A}}\right)$ defines a new dynamical system $\left(R, g, \widehat{m}_{\mathcal{A}}\right)$ and we have the following.

Proposition 6.11. The dynamical system $(R, g)$ admits a unique Equilibrium State associated to $\widehat{\mathcal{B}}_{\mathcal{A}}$, which is the measure $\widehat{m}_{\mathcal{A}}$.

6.2.2. Extension of $\left(R, g, \widehat{m}_{\mathcal{A}}\right)$. The Proposition (B.2) says that a sufficient condition to find an extension of $\left(R, g, \widehat{m}_{\mathcal{A}}\right)$ is that $\int r d \mu_{\mathcal{A}}$ is bounded from above. This part is devoted to the proof of this claim. We claim it is sufficient to prove that

$$
\sum_{n=0}^{+\infty}\left(\sum_{y \in \operatorname{Ant}(n, x)} e^{\mathcal{B}(y)}\right) n e^{-n S}<+\infty
$$

for some $x$ in $F$. Pick one $x$ in $F, y$ in $\operatorname{Ant}(n, x)$. Lemma (A.1) gives two families of sets $\left\{\xi^{s}\left(f^{k}(y)\right)\right\}_{1 \leq k \leq n}$ and $\left\{\xi^{u}\left(f^{k}(y)\right)\right\}_{0 \leq k<n}$, each set containing a ball of radius $\varepsilon$ in the adapted topology, and each set being either wholly inside $R$, or wholly outside. Moreover by Lemma (A.2), there exists an $N$ such that every $\xi^{u}\left(f^{k}(y)\right)$ intersects $f^{-N}(\stackrel{\circ}{R})$ and $\xi^{s}\left(f^{k}(y)\right)$ intersects $f^{N}(\stackrel{\circ}{R})$. Pick $k \leq n-2 N$. Then, there exists an integer $N_{k} \leq N$ and there exists a point $z_{k}(y) \in \xi^{u}(y)=F$ such that 
(i) $f^{j}\left(z_{k}(y)\right) \in \xi^{u}\left(f^{j}(y)\right)$ for all $j \leq k$;

(ii) $f^{k+N}\left(z_{k}(y)\right) \in R, f^{j+N}\left(z_{k}(y)\right) \notin R$ for all $k<j \leq N_{k}$;

(iii) $f^{j+N+N_{k}}\left(z_{k}(y)\right) \in f^{j+N+N_{k}}(y)$ for all $j \leq n-N+N_{k}$.

Lemma 6.12. If $z$ is a point in $\Omega$, there are finitely many $y$ 's in $\operatorname{Ant}(n, x)$ such that $z=z_{k}(y)$ for one $k$. This finite number is uniformly upper bounded in $x$ and $n$.

Proof. If $z=z_{k}(y)=z_{k}\left(y^{\prime}\right)$, then $f^{k}(y)$ and $f^{k}\left(y^{\prime}\right)$ must be $\left(\varepsilon, N+N_{k}\right)$-separated. We denote by $E(\varepsilon, 2 N)$ the largest cardinal of any maximal $(\varepsilon, 2 N)$-separated set. The case $z=z_{k}(y)=z_{k^{\prime}}\left(y^{\prime}\right)$ is impossible because if $k<k^{\prime}$, then $f^{k+N}(z)$ is in $R$ and its orbit doesn't intersect $R$ for $k+N+1$ to $n-1$, and we must have $f^{k^{\prime}+N}(z)$ in $R$. Finally less than $E(\varepsilon, 2 N)$ different $y$ 's can give the same $z=z_{k}(y)$.

If $z=z_{k}(y)$, then $f^{j}(z)$ and $f^{j}(y)$ are close for $0 \leq i \leq k$ and $n-k-N-N_{k} \leq$ $j \leq n$. This proves that there exists an uniform constant $C$ such that

$$
e^{S_{n}(\mathcal{A})(y)} \leq e^{C} e^{S_{n}(\mathcal{A})(z)}
$$

as soon as $z=z_{k}(y)$ for one $k \leq n-2 N$. Thus the case $z=z_{k}(y)=z_{k^{\prime}}(y)$ is impossible for the same reason that $z=z_{k}(y)=z_{k^{\prime}}\left(y^{\prime}\right)$ is impossible. Moreover, if we denote by $\mathcal{Z}$ the set of all points $z=z_{k}(y)$ for one $y$ in $\operatorname{Ant}(n, x)$ and one $k$, all the elements of $\mathcal{Z}$ are in $F$ and have image by $f^{n}$ in $W^{s}(x, R)$. They are preimages of $x$ and $\left\{f^{k}(z)\right\}_{0 \leq k \leq n}$ doesn't intersect $R$ more than $N+2$ times for every $z$. This means that

$$
\mathcal{L}_{\mathcal{A}}(r)(x) \leq\left(e^{C} E(\varepsilon, 2 N) \sum_{k=3}^{N+1} \mathcal{L}_{\mathcal{A}}^{k}\left(\mathbb{1}_{F}\right)(x)\right)+2 N \mathcal{L}_{\mathcal{A}}\left(\mathbb{1}_{F}\right)(x)
$$

As $\mathcal{L}_{\mathcal{A}}\left(\mathbb{1}_{F}\right)(x)$ converges, $\mathcal{L}_{\mathcal{A}}^{k}\left(\mathbb{1}_{F}\right)(x)$ converges for all $k$ and inequality (5) shows that

$$
\mathcal{L}_{\mathcal{A}}(r)(x)<+\infty
$$

We denote by $\nu_{\mathcal{A}}$ the $f$-invariant probability measure on $\Omega$ such that the dynamical system $\left(\Omega, f, \nu_{\mathcal{A}}\right)$ induces the dynamical system $\left(R, g, \widehat{m}_{\mathcal{A}}\right)$. Again we have

$$
\mathcal{P}_{\nu_{\mathcal{A}}}(f, \mathcal{A})=\mathcal{P}_{\mathcal{A}}+\nu_{\mathcal{A}}(R) \log \lambda_{\mathcal{A}}
$$

6.3. Computation of $\lambda_{\mathcal{A}}$. Equality (6) shows that $\lambda_{\mathcal{A}} \leq 1$. We will prove that $\lambda_{\mathcal{A}} \geq 1$, and because of uniqueness of the Equilibrium State for the system $(\Omega, f)$, it will prove that $\nu_{\mathcal{A}}=\mu^{\mathcal{A}}$.

Assume that $\lambda_{\mathcal{A}}<1$; then $\mathcal{L}_{\mathcal{A}}$ is a contraction, and for all $x$ in $F$,

$$
\sum_{n=0}^{+\infty} \mathcal{L}_{\mathcal{A}}^{n}\left(\mathbb{1}_{F}\right)(x)
$$

converges. Pick an $(\varepsilon, n)$-maximal-separated set of points $E_{n}$ and $x$ in $F$. If $y$ is in $E_{n}$, we can find $z_{y}$ such that $z_{y} \in F, f^{N}\left(z_{y}\right)$ and $y$ are $(\varepsilon / 2, n)$-close and $f^{n+2 N}\left(z_{y}\right) \in R$. To $z_{y}$ we associate $y^{\prime}$ such that $\pi_{F} \circ f^{n+2 N}\left(y^{\prime}\right)=x$ and $y^{\prime} \in$ $C_{n+2 N}\left(z_{y}\right)$. We obtain a new set $E_{n}^{\prime}$ such that

$$
e^{-C} \sum_{y \in E_{n}} e^{S_{n}(\mathcal{A})}(y) \leq \sum_{y^{\prime} \in E_{n}^{\prime}} e^{S_{n+2 N}(\mathcal{A})}\left(y^{\prime}\right) \leq e^{C} \sum_{y \in E_{n}} e^{S_{n}(\mathcal{A})}(y) .
$$


If $\left(E_{n}\right)_{n \in \mathbb{N}}$ is a family of $(\varepsilon, n)$-maximal-separated sets, then we have

$$
\begin{aligned}
\sum_{n=0}^{+\infty} \sum_{y \in E_{n}} e^{S_{n}(\mathcal{A})(y)-n \mathcal{P}_{\mathcal{A}}}(y) & \leq e^{C} \sum_{n=0}^{+\infty} \sum_{y^{\prime} \in E_{n}^{\prime}} e^{S_{n+2 N}(\mathcal{A})\left(y^{\prime}\right)-(n+2 N) \mathcal{P}_{\mathcal{A}}}\left(y^{\prime}\right) \\
& \leq e^{C} \sum_{n=0}^{+\infty} \mathcal{L}_{\mathcal{A}}^{n}\left(\mathbb{1}_{F}\right)(x) .
\end{aligned}
$$

We know (see 4]) that there exists a universal constant such that

$$
\frac{1}{C} \leq Z_{n}(\mathcal{A}) e^{-n \mathcal{P}_{\mathcal{A}}} \leq C
$$

where $Z_{n}(\mathcal{A})$ denotes $\sum_{y \in E_{n}} e^{S_{n}(\mathcal{A})(y)}$. Inequality (17) says that $\sum_{n=0}^{+\infty} \mathcal{L}_{\mathcal{A}}^{n}\left(\mathbb{1}_{F}\right)(x)$ cannot converge, and this finally proves that $\lambda_{\mathcal{A}} \geq 1$.

Uniqueness of Equilibrium State gives the following.

Proposition 6.13. The measure $\nu_{\mathcal{A}}$ is the measure $\mu^{\mathcal{A}}$.

\section{Proofs of theorems}

7.1. Proof of Theorem $\mathbf{A}^{\prime}$. We first define a measurable partition subordinate to the stable foliation. The proof of the absolute continuity will not depend on the choice of the partition because of the subordinate condition. We define the partition $\eta^{s}$ in $R$ as $\eta^{s}(x)=W^{s}(x, R)$, and more generally

$$
g^{n} \eta^{s}(x)= \begin{cases}g^{n}\left(\eta^{s}(y)\right) & \text { if there exists } y \text { in } R_{n} \text { such that } g^{n}(y)=x, \\ \{x\} & \text { if not. }\end{cases}
$$

Then Proposition (B.1) yields

Proposition 7.1. There exists a set $\Gamma^{s}$ with full $\mu^{\mathcal{A}}$-measure in $R$ such that for all $x$ in $\Gamma^{s}$ and for all integers $n \in \mathbb{N}$, every non-trivial atom $g^{n} \eta^{s}(y)\left(y \in \eta^{s}(x)\right)$ is such that

$$
\mu_{y}^{\mathcal{A}, s}\left(g^{n} \eta^{s}(y)\right)=\frac{h\left(y_{F}^{n}\right)}{h\left(y_{F}\right)} e^{S_{n}(\mathcal{B})\left(y_{F}^{n}\right)}
$$

where $y_{F} \stackrel{\text { def }}{=} \pi_{F}(y)$ and $y_{F}^{n} \stackrel{\text { def }}{=} \pi_{F} \circ g^{-n}(y)$.

We can now prove Theorem $\mathrm{A}^{\prime}$. We check that for every $(x, y)$ in $\Gamma^{s}$ the two measures $\mu_{x}^{\mathcal{A}, s}$ and $\mu_{y}^{\mathcal{A}, s}$ are equivalent modulo the unstable holonomy.

Lemma 7.2. There exists a universal constant $C$ such that for every $(x, y)$ in $\Gamma^{s}$ if $z_{1}$ and $z_{2}$ are respectively in $\eta^{s}(x)$ and $\eta^{s}(y)$, and $z_{1} \in \eta^{u}\left(z_{2}\right)$ and $g^{n} \eta^{s}\left(z_{1}\right)$ is non trivial for every $n$, then, for all $n$,

$$
\frac{1}{C} \leq \frac{\mu_{y}^{\mathcal{A}, s}\left(g^{n} \eta^{s}\left(z_{2}\right)\right)}{\mu_{x}^{\mathcal{A}, s}\left(g^{n} \eta^{s}\left(z_{1}\right)\right)} \leq C .
$$

Proof. This is a direct result of Proposition (7.1), plus the Hölder continuity property of $\mathcal{B}$ and the continuity of $h$.

Keeping the same notations, we check that the Jacobian is

$$
J\left(z_{1}, z_{2}\right)=\frac{h\left(\pi_{F}(x)\right)}{h\left(\pi_{F}(y)\right)} e^{\left(\sum_{k=1}^{+\infty}\left(\mathcal{A} \circ f^{-k}\left(z_{2}\right)-\mathcal{A} \circ f^{-k}\left(z_{1}\right)\right)+\omega\left(z_{2}\right)-\omega\left(z_{1}\right)\right)} .
$$


The Martingale theorem proves that $J\left(z_{1}, z_{2}\right)=\lim _{n \rightarrow+\infty} \frac{\mu_{y}^{\mathcal{A}, s}\left(g^{n} \eta^{s}\left(z_{2}\right)\right)}{\mu_{x}^{\mathcal{A}, s}\left(g^{n} \eta^{s}\left(z_{1}\right)\right)}$. For $n$ we have

$$
\frac{\mu_{y}^{\mathcal{A}, s}\left(g^{n} \eta^{s}\left(z_{2}\right)\right)}{\mu_{x}^{\mathcal{A}, s}\left(g^{n} \eta^{s}\left(z_{1}\right)\right)}=\frac{h\left(z_{2 F}^{n}\right) h\left(z_{1 F}\right)}{h\left(z_{2 F}\right) h\left(z_{1 F}^{n}\right)} e^{S_{n}(\mathcal{B})\left(z_{2 F}^{n}\right)-S_{n}(\mathcal{B})\left(z_{1 F}^{n}\right)} .
$$

The cocycle property of $\mathcal{B}$ proves that if we set $r=r^{n}\left(z_{1 F}^{n}\right)$,

$$
S_{n}(\mathcal{B})\left(z_{2 F}^{n}\right)-S_{n}(\mathcal{B})\left(z_{1 F}^{n}\right)=\sum_{k=0}^{r-1} \mathcal{A} \circ f^{-k}\left(z_{2 F}^{n}\right)-\mathcal{A} \circ f^{-k}\left(z_{1 F}^{n}\right)+\omega\left(z_{2}\right)-\omega\left(z_{1}\right),
$$

the Hölder properties and the contractions prove that there exists some universal constant $C$ such that

$$
\begin{gathered}
\left|\sum_{k=r / 2}^{r-1} \mathcal{A} \circ f^{k}\left(z_{2 F}^{n}\right)-\mathcal{A} \circ f^{-r+k}\left(z_{2}\right)\right| \leq \frac{C}{\lambda^{r / 2}}, \\
\left|\sum_{k=r / 2}^{r-1} \mathcal{A} \circ f^{k}\left(z_{1 F}^{n}\right)-\mathcal{A} \circ f^{-r+k}\left(z_{1}\right)\right| \leq \frac{C}{\lambda^{r / 2}}, \\
\left|\sum_{k=0}^{r / 2} \mathcal{A} \circ f^{k}\left(z_{2 F}^{n}\right)-\mathcal{A} \circ f^{k}\left(z_{1 F}^{n}\right)\right| \leq \frac{C}{\lambda^{r / 2}} .
\end{gathered}
$$

Inequalities (10) and the continuity of $h$ prove equality (8).

We do the same with $f^{-1}$ and we get a set $\Gamma^{u}$ of full $\mu^{\mathcal{A}}$-measure in $R$. We set $\Gamma=\Gamma^{u} \cap \Gamma^{s}$. For any $x$ and $y$ in $\Gamma, \mu_{x}^{\mathcal{A}, u}$ and $\mu_{y}^{\mathcal{A}, u}$ are equivalent modulo holonomy; hence $\mu_{x}^{\mathcal{A}, u}$ is equivalent to $m_{\mathcal{A}}$ for every $x$ in $F$. This proves that $\mu^{\mathcal{A}}$ has a local product structure in $R$ and then in all $\Omega$.

By [12], we know the existence of a set $\Delta$ of full $\mu^{\mathcal{A}}$-measure of points such that $\mu_{x}^{\mathcal{A}, s}$ and $\mu_{x}^{\mathcal{A}, u}$ have pointwise dimensions equal to $\delta^{s}$ and $\delta^{u}$. Pick any point $x$ in $\Delta \cap \Gamma$. Because of the continuity of $x \mapsto E^{u}(x)$ and $x \mapsto E^{s}(x)$, there exists a universal constant $C$ such that for every $\rho$ small enough we get

$$
\left[B^{u}\left(x, \frac{\rho}{C}\right) ; B^{s}\left(x, \frac{\rho}{C}\right)\right] \subset B(x, \rho) \subset\left[B^{u}(x, C \rho) ; B^{s}(x, C \rho)\right],
$$

and the absolute continuity of conditional measures yields

$$
\begin{aligned}
\frac{1}{C^{\prime}} \mu^{\mathcal{A}, u}\left(B^{u}(x, \rho)\right) \cdot \mu^{\mathcal{A}, s}\left(B^{s}(x, \rho)\right) & \leq \mu^{\mathcal{A}}\left(\left[B^{u}(x, \rho) ; B^{s}(x, \rho)\right]\right) \\
& \leq C^{\prime} \mu^{\mathcal{A}, u}\left(B^{u}(x, \rho)\right) \cdot \mu^{\mathcal{A}, s}\left(B^{s}(x, \rho)\right),
\end{aligned}
$$

for some universal constant $C^{\prime}$. Then (11) and (12) finish proof of Theorem $\mathrm{A}^{\prime}$.

7.2. Proof of Theorem A. We first recall a result from $[5]$ in the case of invariant transversal measure.

Lemma 7.3. Suppose that $\mathcal{E}$ is a subfamily of transversals to the stable foliation such that $\Omega=\left\{W^{s}(x), x \in \bigcup_{K \in \mathcal{E}}\right.$ int $\left.\Phi\left(K \times D^{n}\right)\right\}$. Then the map

$$
\left\{\mu_{K}\right\}_{K} \text { transversal } \longrightarrow\left\{\mu_{K}\right\}_{K \in \mathcal{E}}
$$

is a bijection between $W^{s}$-invariant measures and a family of measures on $\{K \in \mathcal{E}\}$ satisfying the required conditions on these sets. 
If $x$ is in $\Omega$, then $W^{s}(x) \cap \operatorname{int}(F) \neq \emptyset$, and $\operatorname{int}(F) \subset \operatorname{int}(R)$. This shows that to prove existence and uniqueness of $\left\{\mu_{K}\right\}$ it is sufficient to prove existence and uniqueness of such a measure on the unstable leaf $F$.

Then we check that $\mu_{\mathcal{A}}$ is the unique measure satisfying the conditions.

The two conditions prove that if such a measure exists, it must be a conformal measure for the system $\left(F, g_{F}\right)$ with Jacobian $e^{-S_{r(x)}(\mathcal{A})(x)+r(x) \mathcal{P}_{\mathcal{A}}-\omega(x)}$. This yields that the measure must be $\mu_{\mathcal{A}}$. We check existence now. Pick $A$ and $A^{\prime}$ two Borel sets, with a one-to-one Borel map $\pi_{A, A^{\prime}}$ between them. We first assume that $A \subset F_{\infty}$ and $A^{\prime} \subset F_{\infty}$. Set $A=\bigsqcup_{n \in \mathbb{N}^{*}} A_{n}$ such that $x \in A_{n}$ if and only if $n$ is the first integer such that $f^{n}(x) \in R$ and $f^{n} \circ \pi_{A, A^{\prime}}(x) \in W^{s}\left(f^{n}(x), R\right)$. Cut any $A_{n}$ in countable $A_{n}^{m}$ such that, on each $A_{n}^{m}, f^{n}$ corresponds to a map $g^{k_{n, m}}$ and, on each $A_{n}^{\prime m}, f^{n}$ corresponds to a map $g^{k_{n, m}^{\prime}}$. Hence $g_{F}^{k_{n, m}}\left(A_{n}^{m}\right)=g_{F}^{k_{n, m}^{\prime}}\left(A_{n}^{\prime m}\right)$, the cocycle property of $\mathcal{B}$, and the fact that $\mu_{\mathcal{A}}$ is conformal prove that

$$
\forall(n, m) \in \mathbb{N}^{*} \times \mathbb{N} \quad \mu_{\mathcal{A}}\left({A^{\prime}}_{n}^{m}\right)=\int_{A_{n}^{m}} e^{w\left(\pi_{A, A^{\prime}}(x), x\right)} d \mu_{\mathcal{A}} .
$$

This proves that $\mu_{\mathcal{A}}$ satisfies conditions of Theorem A. Moreover, $\mu_{\mathcal{A}}$ is equivalent to $m_{\mathcal{A}}$ which is equivalent to $\mu_{x}^{\mathcal{A}, u}$ for any $x \in F$ by Theorem $\mathrm{A}^{\prime}$.

\section{Appendix A. Some facts about Axiom A}

We give here some general facts in the Axiom-A theory. In this part, we assume we have a cover of the basic set $\Omega$ by some Markov partition, denoted by $\mathcal{R}=\left\{R_{i}\right\}$. The finite union of unstable (resp. stable) boundaries will be denoted by $\partial^{u} \mathcal{R}$ (resp. $\left.\partial^{s} \mathcal{R}\right)$. The map $x \mapsto d^{s}\left(x, \partial^{u} \mathcal{R}\right)$, where $d^{s}$ denotes the canonical metric on the stable leaves, is continuous. Then for each $i$, there exists some constant $d_{i}>0$ such that, for all $x \in R_{i}, W^{s}\left(x, R_{i}\right)$ contains a ball of radius bigger than $d_{i}$. As we have finitely many rectangles, we have $0<\underline{d}=\min _{i} d_{i}$.

Lemma A.1. Assume $x$ is in $R_{0}, f^{n}(x) \in R_{0}$ and $f^{k}(x) \notin R_{0}$ for every $1 \leq k \leq$ $n-1$. Then there exists a family of sets $\left(\xi^{s}\left(f^{k}(x)\right)\right)_{1 \leq k \leq n}$ such that

(i) $\xi^{s}\left(f^{n}(x)\right)=W^{s}\left(f^{n}(x), R_{0}\right)$;

(ii) for any $k$ in $[1, n-1] \xi^{s}\left(f^{k}(x)\right) \subset W_{l o c}^{s}\left(f^{k}(x)\right)$, and $\xi^{s}\left(f^{k}(x)\right) \cap R_{0}=\emptyset$;

(iii) for any $k, f\left(\xi^{s}\left(f^{k}(x)\right)\right) \subset \xi^{s}\left(f^{k+1}(x)\right)$.

Moreover, there exists an uniform constant $\varepsilon=\varepsilon(\mathcal{R})$ such that

$$
\xi^{s}\left(f^{k}(x)\right) \supset B^{s}\left(y_{k}, \varepsilon\right) \ni f^{k}(x)
$$

for some $y_{k}$.

Proof. Pick one $x$ and one $n$. We first set $\xi^{s}\left(f^{n}(y)\right)=W^{s}\left(f^{n}(x), R_{0}\right)$. Assume we have defined $\xi^{s}\left(f^{k}(x)\right)$ and that its boundary is included in $\partial^{u} \mathcal{R}$. We define $\xi^{s}\left(f^{k-1}(x)\right)$ in the following way: $f^{-1}\left(\xi^{s}\left(f^{k}(x)\right)\right)$ contains $f^{k-1}(x)$ which is outside of $R_{0}$, and its boundary is again in $\partial^{u} \mathcal{R}$. If the interior of $f^{-1}\left(\xi^{s}\left(f^{k}(x)\right)\right)$ (for the $W^{s}$-topology) doesn't intersect with $\partial^{u} \mathcal{R}$, then $f^{-1}\left(\xi^{s}\left(f^{k}(x)\right)\right) \cap \stackrel{\circ}{R_{0}}=\emptyset$, and we set $\xi^{s}\left(f^{k-1}(x)\right)=f^{-1}\left(\xi^{s}\left(f^{k}(x)\right)\right)$. Otherwise, the Markov property of $\mathcal{R}$ proves that we can find an open subset of $f^{-1}\left(\xi^{s}\left(f^{k}(x)\right)\right)$ (in the $W^{s}$-topology) with boundary in $\partial^{u} \mathcal{R}$ which contains $f^{k-1}(x)$ and doesn't intersect with $R_{0}$. This set defines $\xi^{s}\left(f^{k-1}(x)\right)$. In each case, $\xi^{s}\left(f^{k-1}(x)\right)$ contains a ball in the stable foliation of radius $\underline{d} / 2$.

Remark. We have also a family of set $\left(\xi^{u}\left(f^{k}(y)\right)\right)_{1 \leq k \leq n}$ with symmetric properties. 
We formulate now in a different way the mixing property.

Lemma A.2. Let $\varepsilon$ be an expansiveness constant. There exists an integer $N$ such that for every $x \in \Omega W_{\varepsilon}^{u}(x) \cap f^{-N}\left(\stackrel{\circ}{R}_{0}\right) \neq \emptyset$.

Proof. Because $\stackrel{\circ}{R}_{0}$ is an open set, it contains a periodic point $x_{0}$. Let $p_{0}$ be $x_{0}$ 's period. As $W^{s}\left(x_{0}\right)$ is dense in $\Omega$, for every $x$ in $\Omega$, there exists some $n$, such that $W_{\varepsilon}^{u}(x) \cap f^{-n p_{0}} W^{s}\left(x_{0}, \stackrel{\circ}{R_{0}}\right) \neq \emptyset$. Moreover, if $W_{\varepsilon}^{u}(x) \cap f^{-n p_{0}} W^{s}\left(x_{0}, \stackrel{\circ}{R_{0}}\right) \neq$ $\emptyset$, then there is an open set $U$ containing $x$ such that for all $y$ in $U W_{\varepsilon}^{u}(y) \cap$ $f^{-n p_{0}} W^{s}\left(x_{0}, \stackrel{\circ}{R}_{0}\right) \neq \emptyset$. By compactness we can find a finite number of such open sets, and a finite maximal $N$.

Remark. If $N$ is taken large enough, we know that $f^{N}\left(W_{\varepsilon}^{u}(x)\right) \cap \stackrel{\circ}{R}_{0}$ is an unstable leaf of $\stackrel{\circ}{R}_{0}$. In the same way, if $N$ is taken sufficiently large, $W_{\varepsilon}^{s}(x) \cap f^{N}\left(\stackrel{\circ}{R}_{0}\right) \neq \emptyset$ for all $x$ and $f^{-N}\left(W_{\varepsilon}^{s}(x)\right) \cap \stackrel{\circ}{R}_{0}$ is a stable leaf of $\stackrel{\circ}{R}_{0}$.

QUESTION: In this part only we seem to be forced to assume existence of a cover by rectangles with the Markov property. This could be an obstacle to extending this method to the non-uniform hyperbolic case. Is there a geometrical way to prove that if we have just one rectangle with the Markov property, then the set $\Omega \backslash \bigcup_{n>0} f^{-n}\left(\partial^{u} R\right)$ must be a union of open sets containing balls of big radius in the $W^{s}$-topology?

\section{Appendix B. Some facts about extensions}

We give here some general facts about extensions. We first recall that if $(Y, \Psi, \nu)$ is a dynamical system, the measure $\nu$ is said to be conformal with Jacobian $J$, if for all Borel set $B$ such that $\Psi_{\mid B}$ is one-to-one,

$$
\nu(\Psi(B))=\int_{B} J d \nu .
$$

The next result gives a relation between the measure $\nu$ of a system $(Y, \Psi, \nu)$ and the conditional measures along the fibers of its natural extension.

Proposition B.1. Let $(Y, \Psi, \nu)$ be a dynamical system and $(X, \Phi, \mu)$ its natural extension. Let $\Pi$ be the canonical map from $X$ onto $Y$. Assume there exists a countable partition (in the sense of $\nu$ ) $\left(Y_{n}\right)_{n \in \mathbb{N}}$ of $Y$ such that for every $n \Psi\left(Y_{n}\right)=$ $Y$. Assume it is a generating partition

$$
\bigvee_{n=0}^{+\infty} \Psi^{-n}\left(Y_{n}\left(\Psi^{n}(y)\right)\right)=\{y\} \quad \nu \text {-a.e. }
$$

and each fiber in $X, X_{y} \stackrel{\text { def }}{=}\{x \in X, \Pi(x)=y\}$, has a countable number of images of fibers by $\Phi$. Then the partition of $X$ in fibers is measurable and if $\mu_{x}$ denotes its unique system of conditional measures, then $\nu$ is conformal with Jacobian

$$
\frac{1}{\mu_{\Psi(y)}\left(\Phi\left(\Pi^{-1}(y)\right)\right)} .
$$

We give now another result which says when a system can be seen as an induced sub-system. 
Proposition B.2. Let $(Y, \Psi, \nu)$ be a dynamical system with $\nu$ a $\Psi$-invariant ergodic Borel probability measure. Assume that

(1) $Y \subset X$;

(2) $\Psi$ is almost everywhere the first return map in $Y$ associated to the iteration of an inversible map $\Phi$ from $X$ onto itself;

(3) If $r$ is the first return time map $\left(\Psi(x)=\Phi^{r(x)}(x)\right)$, then $\int r d \nu<+\infty$.

Then there exists a $\Phi$-invariant ergodic Borel probability measure such that $(X, \Phi, \mu)$ induces by the first return map the system $(Y, \Psi, \nu)$.

Proof. We give here just the sketch of the proof. For a complete proof, see [7].

Denote by $l \stackrel{\text { def }}{=} \frac{1}{\int r d \nu}$. Pick any Borel set in $X$, and any integer $k$. Set

$$
A_{k} \stackrel{\text { def }}{=}\left\{y \in A \text { such that } \Phi^{-k}(y) \in Y \text { and } \Phi^{-j}(y) \notin Y \forall j<k\right\}
$$

and define

$$
\mu(A) \stackrel{\text { def }}{=} l \times\left(\sum_{k=0}^{+\infty} \nu\left(\Phi^{-k}\left(A_{k}\right)\right)\right) .
$$

Just check that $\mu$ satifies the required properties.

\section{Appendix C. IOnEscu-Tulcea And Marinescu's theorem}

We recall here Ionescu-Tulcea and Marinescu's theorem, and some useful lemmas. Some complete proofs can be found in [10] or [6].

Ionescu-Tulcea \& Marinescu Theorem. Let $\left(\mathcal{V},\|\|_{\mathcal{V}}\right)$ and $\left(\mathcal{U},\|\|_{\mathcal{U}}\right)$ be two $\mathbb{C}$ Banach spaces such that $\mathcal{V} \subset \mathcal{U}$. We assume that

(i) if $\left(\varphi_{n}\right)_{n \in \mathbb{N}}$ is a sequence of functions in $\mathcal{V}$ which converges in $\left(\mathcal{U},\|\|_{\mathcal{U}}\right)$ to a function $\varphi$ and if, for all $n \in \mathbb{N},\left\|\varphi_{n}\right\|_{\mathcal{V}} \leq C$, then $\varphi \in \mathcal{V}$ and $\|\varphi\|_{\mathcal{V}} \leq C$,

and $\Phi$ is an operator from $\mathcal{U}$ to itself such that

(ii) $\Phi$ leaves $\mathcal{V}$ invariant and is bounded for \|\|$_{\mathcal{V}}$;

(iii) $\sup _{n}\left\{\left\|\Phi^{n}(\varphi)\right\|_{\mathcal{U}}, \varphi \in \mathcal{V},\|\varphi\|_{\mathcal{U}} \leq 1\right\} \leq M_{S}<\infty$;

(iv) there exist an integer $n_{0}$ and two constants $0<a<1$ and $0 \leq b<+\infty$ such that for all $\varphi \in \mathcal{V}$ we have $\left\|\Phi^{n_{0}}(\varphi)\right\|_{\mathcal{V}} \leq a\|\varphi\|_{\mathcal{V}}+b\|\varphi\|_{\mathcal{U}}$;

(v) if $\mathcal{X}$ is a bounded subset of $(\mathcal{V},\|\| \mathcal{V})$, then $\Phi^{n_{0}}(\mathcal{X})$ has compact closure in $\left(\mathcal{U},\|\|_{\mathcal{U}}\right)$.

Then $\Phi$ has a finite number of eigenvalues of norm $1: \lambda_{1}, \ldots, \lambda_{p}$ and $\Phi$ can be written $\Phi=\sum_{i=1}^{p} \lambda_{i} \Phi_{i}+\Psi$, where the $\Phi_{i}$ are linear bounded operators from $\mathcal{V}$ to $\Phi(\mathcal{V})$ of finite dimension image contained in $\mathcal{V}$, and where $\Psi$ is a linear bounded operator with spectral radius $\rho(\Psi)<1$ in $(\mathcal{V},\|\| \mathcal{V})$.

Moreover the following hold: $\Phi_{i} \cdot \Phi_{j}=\Phi_{j} . \Phi_{i}=0$ for all $i \neq j, \Phi_{i} . \Phi_{i}=\Phi_{i}$ for all $i$, and $\Phi_{i} . \Psi=\Psi \cdot \Phi_{i}=0$ for all $i$.

Remark. In particular, we will get, for all $n, \Phi^{n}=\sum_{i=1}^{p} \lambda_{i}^{n} \Phi_{i}+\Psi^{n}$.

Under the same assumptions we get the three following results.

Lemma C.1. There exists $L \in \mathbb{R}_{+}$such that for all $m \in \mathbb{N}$ and for all $\varphi \in \mathcal{V}$ we have $\left\|\Phi^{m n_{0}}(\varphi)\right\|_{\mathcal{V}} \leq a^{m}\|(\varphi)\|_{\mathcal{V}}+L\|(\varphi)\|_{\mathcal{U}}$.

Lemma C.2. The family $\left(\left\|\Phi^{m}\right\|_{\mathcal{V}}\right)_{m}$ is uniformly bounded. 
Lemma C.3. For all $\varphi \in \mathcal{V}$ there exists $\bar{\varphi} \in \mathcal{V}$ such that

$$
\lim _{n \rightarrow+\infty}\left\|\frac{1}{n} \sum_{k=0}^{n-1} \Phi^{k+n_{0}}(\varphi)-\bar{\varphi}\right\|_{\mathcal{U}}=0 .
$$

\section{ACKNOWLEDGMENTS}

This work was done at the Université Paris-Sud (Orsay/ France), when I was a Ph.D. student under the direction of Philippe Thieullen. I would like to thank him for all the helpful advice he gave me. I also thank J.C. Léger, for correcting my English.

\section{REFERENCES}

1. M. Babillot and F. Ledrappier, Geodesic paths and horocycle flow on abelian covers, Centre de Mathématiques de l'École Polytechnique.

2. L. Barreira, Y. Pesin, and J. Schmeling, Dimension of hyperbolic measures - A proof of the Eckmann-Ruelle conjecture, Weierstraß-Institut für Angewandte Analysis und Stochastik, 1996.

3. R. Bowen, Some Systems with Unique Equilibrium States, Mathematical Systems Theory 8 (1974), no. 3, 193-202. MR 53:3257

4. - Equilibrium States and the Ergodic Theory of Anosov Diffeomorphisms, Lecture Notes in Math., vol. 470, Springer-Verlag, 1975. MR 56:1364

5. R. Bowen and B. Marcus, Unique Ergodicity for Horocycle Foliations, Israel Journal of Mathematics 26 (1977), no. 1, 43-67. MR 56:9594

6. A. Broise, F. Dal'bo, and M. Peigné, Méthode de opérateurs de transferts : Transformations dilatantes de l'intervalle et dénombrement de géodésiques fermées.

7. Yael Naim Dowker, Finite and $\sigma$-finite invariant measures, Annals of Mathematics 54 (1951), no. 3, 595-608. MR 13:543a

8. Nicolai T.A. Haydn, Canonical product structure of equilibrium states, Random and Computational Dynamics 2 (1994), no. 1, 79-96. MR 95c:58133

9. N.T.A. Haydn and D. Ruelle, Equivalence of Gibbs and Equilibrium States for Homeomorphisms Satisfying Expansiveness and Specification, Commun. Math. Phys. 148 (1992), 155167. MR 93h:58084

10. C.T. Ionescu Tulcea and G. Marinescu, Théorie ergodique pour des classes d'opérations non complètement continues, Annals of Mathematics 52 (1950), no. 1, 140-147. MR 12:266g

11. F. Ledrappier and L.-S. Young, The metric entropy of diffeomorphisms Part I: Characterization of measures satisfying Pesin's entropy formula, Annals of Mathematics 122 (1985), 509-539. MR 87i:58101a

12. and dimension, Annals of Mathematics 122 (1985), 540-574. MR 87i:58101b

13. V.A. Rohlin, On the fundamental ideas of measure theory, A.M.S.Translation 10 (1962), 1-52. MR 11:18f, MR 13:924e

14. Walter Rudin, Real and Complex Analysis, Third edition, McGraw-Hill Book Company, 1987. MR 88k:00002

15. D. Ruelle, Thermodynamic formalism for maps satisfying positive expansiveness and specification, Nonlinearity 5 (1992), 1223-1236. MR 94a:58115

Laboratoire de Mathématique et Applications des Mathématiques, Université de Bretagne-Sud, 1, Rue de la Loi, 56000 Vannes, France

E-mail address: Renaud.Le-Plaideur@univ-ubs.fr 